TRANSACTIONS OF THE

AMERICAN MATHEMATICAL SOCIETY

Volume 356, Number 10, Pages 3899-3937

S 0002-9947(04)03530-5

Article electronically published on February 4, 2004

\title{
SURFACE SUPERCONDUCTIVITY IN 3 DIMENSIONS
}

\author{
XING-BIN PAN
}

\begin{abstract}
We study the Ginzburg-Landau system for a superconductor occupying a 3-dimensional bounded domain, and improve the estimate of the upper critical field $H_{C_{3}}$ obtained by K. Lu and X. Pan in J. Diff. Eqns., 168 (2000), 386-452. We also analyze the behavior of the order parameters. We show that, under an applied magnetic field lying below and not far from $H_{C_{3}}$, order parameters concentrate in a vicinity of a sheath of the surface that is tangential to the applied field, and exponentially decay both in the normal and tangential directions away from the sheath in the $L^{2}$ sense. As the applied field decreases further but keeps in between and away from $H_{C_{2}}$ and $H_{C_{3}}$, the superconducting sheath expands but does not cover the entire surface, and superconductivity at the surface portion orthogonal to the applied field is always very weak. This phenomenon is significantly different to the surface superconductivity on a cylinder of infinite height studied by X. Pan in Comm. Math. Phys., 228 (2002), 327-370, where under an axial applied field lying in-between $H_{C_{2}}$ and $H_{C_{3}}$ the entire surface is in the superconducting state.
\end{abstract}

\section{§1. INTRODUCTION}

Surface nucleation of superconductivity in applied magnetic fields decreasing from the upper critical field $H_{C_{3}}$ has been studied based on the Ginzburg-Landau theory by many authors; see, for instance, [SdG], dG], [SST] and $[\mathrm{T}]$ for early references, and [C], [BPT], [GP], [BS], [DFS], [LP1], [LP2], [LP3], [LP4], [HM1], HM2 and $\mathrm{HP}$ for recent mathematical research. It is the estimate of $H_{C_{3}}$ and the location of nucleation that we are most interested in.

In [LP1], LP2], [LP3] and [HP] we studied the surface nucleation phenomena for a cylindrical superconductor of infinite height placed in an axial applied magnetic field $\mathcal{H}$. Then the Ginzburg-Landau system is reduced to a two-dimensional problem on the cross section $D$ of the cylinder, and hence this case is called a 2 -dimensional superconductivity. It has been proved in [HP] that, for large $\kappa$,

$$
H_{C_{3}}(\kappa)=\frac{\kappa}{\beta_{0}}+\frac{C_{1}}{\beta_{0}^{3 / 2}} \kappa_{\max }+O\left(\kappa^{-1 / 3}\right),
$$

where $\kappa$ is the Ginzburg-Landau parameter given by the ratio of the penetration depth and the coherence length of the superconductor, $\beta_{0}$ is the lowest eigenvalue of the Schrödinger operator with the unit magnetic field on the half plane, $C_{1}$ is a universal constant (see Section 2 for the definition of $\beta_{0}$ and $C_{1}$ ), and $\kappa_{\max }$ is the maximum value of the curvature of the boundary curve $\partial D$ of the cross section

Received by the editors October 12, 2001 and, in revised form, May 19, 2003.

2000 Mathematics Subject Classification. Primary 35Q55, 82D55.

Key words and phrases. Ginzburg-Landau system, superconductivity, nucleation, upper critical field, Schrödinger operator with a magnetic field. 
$D$. Moreover, when the applied field is spatially homogeneous and decreases from $H_{C_{3}}$, a superconducting sheath nucleates on a subset of the lateral surface where the curvature of $\partial D$ is maximal. In [P1] we further showed that, if the applied field lies in-between and keeps away from $H_{C_{2}}$ and $H_{C_{3}}$, the sample is in the surface superconducting state, namely, superconductivity persists uniformly at a thin sheath surrounding the entire lateral surface of the sample, while the interior of the sample remains close to the normal state. For relative works see, for instance, $[\mathrm{C}], \mathrm{BPT}$, [GP, [BS], [HM1], [SS], [A1].

Our understanding of surface nucleation on a 3-dimensional bounded superconductor is far from being complete. Before stating our main questions and results, we recall that, according to the Ginzburg-Landau theory, superconductivity is described by a complex-valued function $\psi$ (order parameter) and a real vector field $\mathcal{A}$ (magnetic potential), and $(\psi, \mathcal{A})$ is a minimizer of the Ginzburg-Landau functional. Let us consider a homogeneous applied field $\mathcal{H}=\sigma \mathbf{h}$ where $\mathbf{h}$ is a constant unit vector and $\sigma$ is a parameter, and set $\mathcal{A}=\sigma \mathbf{A}$. With proper scaling, we may rewrite the Ginzburg-Landau functional as follows ([GL], [dG], [CHO], [DGP]):

$$
\mathcal{E}[\psi, \mathbf{A}]=\int_{\Omega}\left\{\left|\nabla_{\kappa \sigma \mathbf{A}} \psi\right|^{2}-\kappa^{2}|\psi|^{2}+\frac{\kappa^{2}}{2}|\psi|^{4}\right\} d x+\kappa^{2} \sigma^{2} \int_{\mathbb{R}^{3}}|\operatorname{curl} \mathbf{A}-\mathbf{h}|^{2} d x,
$$

where $\nabla_{\kappa \sigma \mathbf{A}} \psi=\nabla \psi-i \kappa \sigma \mathbf{A} \psi, i=\sqrt{-1}$. Then we write the Ginzburg-Landau system for $(\psi, \mathbf{A})$ (the Euler equation of the functional $\mathcal{E}$ ) as follows:

$$
\begin{cases}-\nabla_{\kappa \sigma \mathbf{A}}^{2} \psi=\kappa^{2}\left(1-|\psi|^{2}\right) \psi & \text { in } \Omega, \\ \operatorname{curl}^{2} \mathbf{A}=-\frac{i}{2 \kappa \sigma}(\bar{\psi} \nabla \psi-\psi \nabla \bar{\psi})-|\psi|^{2} \mathbf{A} & \text { in } \Omega, \\ \operatorname{curl}^{2} \mathbf{A}=\mathbf{0} & \text { in } \mathbb{R}^{3} \backslash \bar{\Omega}, \\ \left(\nabla_{\kappa \sigma \mathbf{A}} \psi\right) \cdot \nu=0, \quad[\nu \times \mathbf{A}]=0, \quad[\nu \times \operatorname{curl} \mathbf{A}]=\mathbf{0} & \text { on } \partial \Omega, \\ \operatorname{curl} \mathbf{A} \rightarrow \mathbf{h} \quad \text { as }|x| \rightarrow \infty, & \end{cases}
$$

where $\nabla_{\mathbf{A}}^{2} \psi=(\nabla-i \mathbf{A})^{2} \psi=\Delta \psi-i(2 \mathbf{A} \cdot \nabla \psi+\psi \operatorname{div} \mathbf{A})-|\mathbf{A}|^{2} \psi, \nu$ is the unit outer normal vector at the boundary of $\Omega$, and [ · ] denotes the jump in the enclosed quantity across $\partial \Omega$. Note that in the above system the unit of length is the penetration depth. A (global) minimizer of the Ginzburg-Landau functional is called a minimal solution of (1.2). Throughout this paper we assume that $\Omega$ is a bounded, smooth (say, $C^{4}$ ) and simply-connected domain in $\mathbb{R}^{3}$. Let $\mathbf{F}_{\mathbf{h}}$ be a smooth vector field on $\mathbb{R}^{3}$ such that

$$
\operatorname{curl} \mathbf{F}_{\mathbf{h}}=\mathbf{h} \text { and } \operatorname{div} \mathbf{F}_{\mathbf{h}}=0 \text { in } \mathbb{R}^{3} .
$$

For a given $\mathbf{h}, \mathbf{F}_{\mathbf{h}}$ is unique up to a harmonic gradient field (gradient of a harmonic function $)\left(0, \mathbf{F}_{\mathbf{h}}\right)$ is a trivial solution of (1.2) and corresponds to the normal state. A mathematical definition of the upper critical field $H_{C_{3}}$ has been given in [LP3], LP4]:

$$
H_{C_{3}}(\mathbf{h}, \kappa)=\inf \left\{\sigma>0:\left(0, \mathbf{F}_{\mathbf{h}}\right) \text { is a minimizer of } \mathcal{E}\right\} .
$$

The nucleation of superconductivity can be described by the fact that, as an applied field decreases from $H_{C_{3}},(1.2)$ has a nontrivial minimal solution $(\psi, \mathbf{A})$ with $|\psi|$ being small; see [LP3], [LP4].

\footnotetext{
${ }^{1}$ Hence the eigenvalues of the operators involving $\mathbf{F}_{\mathbf{h}}$ discussed in this paper are independent of the choice of $\mathbf{F}_{\mathbf{h}}$.
} 
In [LP4] we proved that, for a 3-dimensional bounded, smooth and simplyconnected domain,

$$
\frac{\kappa}{\beta_{0}}-C \kappa^{1 / 3} \leq H_{C_{3}}(\mathbf{h}, \kappa)=\frac{\kappa}{\beta_{0}}+o(\kappa),
$$

and showed that, as the applied field decreases from $H_{C_{3}}(\mathbf{h}, \kappa)$, a superconducting sheath nucleates at the tangential set $(\partial \Omega)_{\mathbf{h}}$, which is a subset of the surface that is tangential to the applied field?:

$$
(\partial \Omega)_{\mathbf{h}}=\{x \in \partial \Omega: \mathbf{h} \cdot \nu(x)=0\} .
$$

Note that if the applied field is very close to $H_{C_{3}}$, the nucleation set, to which superconductivity is confined, is a proper subset of $(\partial \Omega)_{\mathbf{h}}$. As the applied field decreases further, the order parameters will grow up and the superconducting layer will expand; see [SST], p. 87. Thus we want to ask

Question 1. Find the geometric characterization of the nucleation set, and find the profile of the superconducting layer when the applied field is below and close to $H_{C_{3}}$.

Question 2. Find the concentration behavior of order parameters when the applied field lies in-between and keeps away from $H_{C_{2}}$ and $H_{C_{3}}$.

In this paper we are able to give an answer to Question 2, and give some preparation for solving Question 1. In order to answer Question 1, we have to improve the estimate (1.4) by catching the next higher order term in the expansion, which will enable us to locate the nucleation set more precisely. For this purpose we need the following notation. For $x \in \partial \Omega$, let $\kappa_{1}(x)$ and $\kappa_{2}(x)$ be the principal curvatures of $\partial \Omega$ at $x$, and let $\theta_{\mathbf{h}}=\theta_{\mathbf{h}}(x)$ be the angle between $\mathbf{h}$ and the principal direction corresponding to $\kappa_{1}(x)$. We define a function $P(x)$ on $\partial \Omega$ by

$$
P(x)=\left[\kappa_{1}^{2} \cos ^{2} \theta_{\mathbf{h}}+\kappa_{2}^{2} \sin ^{2} \theta_{\mathbf{h}}-\alpha_{0}\left(\kappa_{1}-\kappa_{2}\right)^{2} \cos ^{2} \theta_{\mathbf{h}} \sin ^{2} \theta_{\mathbf{h}}\right]^{1 / 3},
$$

where the constant $\alpha_{0}$ will be given in (2.4) in Section 2, and $0<\alpha_{0}<1$. Let

$$
\begin{aligned}
& P_{\min }=\min _{x \in(\partial \Omega)_{\mathbf{h}}} P(x), \\
& \mathcal{P}=\left\{x \in(\partial \Omega)_{\mathbf{h}}: P(x)=P_{\min }\right\} .
\end{aligned}
$$

Let $\lambda_{0}$ be the lowest eigenvalue of (2.14) in Section 2, and let

$$
C_{2}=\lambda_{0}\left(1-\alpha_{0}\right)^{1 / 3} \text {. }
$$

We have:

Theorem 1. Let $\Omega$ be a bounded, smooth and simply-connected domain in $\mathbb{R}^{3}$ and $\mathbf{h}$ a unit vector. We have, for large $\kappa$ :

$$
\frac{\kappa}{\beta_{0}}-\frac{C_{2}}{\beta_{0}^{5 / 3}} P_{\min } \kappa^{1 / 3}+O\left(\kappa^{2 / 9}\right) \leq H_{C_{3}}(\mathbf{h}, \kappa) \leq \frac{\kappa}{\beta_{0}}+M \kappa^{1 / 2},
$$

where $M$ is a constant independent of $\mathbf{h}$ and $\kappa$.

\footnotetext{
${ }^{2}$ The situation will be different for samples with nonsmooth surface; see [JRS], [J] and [P2]
} 
In the proof of Theorem 1, the eigenvalue estimate and classification of the eigenfunctions of the Schrödinger operator with a nondegenerately vanishing magnetic field established by Montgomery [M], Helffer [H] and Pan-Kwek [PK] play an important role.

The right side estimate in (1.7) should be improved. In fact, we have the following

Conjecture. Let $\Omega$ be a bounded, smooth and simply-connected domain in $\mathbb{R}^{3}$ and $\mathbf{h}$ a unit vector. Then

$$
H_{C_{3}}(\mathbf{h}, \kappa)=\frac{\kappa}{\beta_{0}}-\frac{C_{2}}{\beta_{0}^{5 / 3}} P_{\min } \kappa^{1 / 3}+O\left(\kappa^{2 / 9}\right) .
$$

The estimate (1.8) will enable us to answer Question 1 by proving that, as the applied field decreases from $H_{C_{3}}$, superconductivity nucleates first at the subset $\mathcal{P}$ of $\partial \Omega$, where the applied field is tangential to $\partial \Omega$, and the function $P(x)$ is minimal 3

With only (1.7) in hand, however, we are still able to investigate the behavior of the order parameters for a superconductor in an applied field which is below $H_{C_{3}}$ by a gap of scale $\kappa^{1 / 2}$, and give a satisfactory answer to Question 2; see Theorem 2 . We use the following notation: Given a unit vector $\mathbf{h}$ and $x \in \partial \Omega$, let $\varphi_{\mathbf{h}}(x)$ denote the angle between $\mathbf{h}$ and the outer normal vector $\nu(x), 0 \leq \varphi_{\mathbf{h}}(x) \leq \pi$. Note that $\varphi_{\mathbf{h}}(x)=\pi / 2$ on $(\partial \Omega)_{\mathbf{h}}$. We can extend $\varphi_{\mathbf{h}}(x)$ to a continuous function on $\bar{\Omega}$; see Remark 2.4 in Section 2. Given $\delta>0$, let

$$
\Omega(\delta)=\{x \in \bar{\Omega}: \operatorname{dist}(x, \partial \Omega) \leq \delta\} .
$$

Using Theorem 1 and adapting the methods from [HP] involving Agmon's idea $[\mathrm{A}]$ and the techniques developed by Helffer $[\mathrm{H}]$, we can prove the following.

Theorem 2. Let $\Omega$ be a bounded, smooth and simply-connected domain in $\mathbb{R}^{3}$ and $\mathbf{h}$ a unit vector. Under the applied field $\mathcal{H}=\sigma \mathbf{h}$, the order parameters $\psi$ have the following concentration behavior for large $\kappa$ :

(1) If $\sigma=H_{C_{3}}(\mathbf{h}, \kappa)-a_{1} \kappa^{1 / 2}$ where $a_{1}>0$, there exist positive constants $\eta, \gamma_{1}$ and $M_{1}$, such that for large $\kappa$,

$$
\int_{\Omega \backslash \mathcal{S}_{\kappa}(\mathbf{h})}|\psi|^{2} d x \leq M_{1} \exp \left(-\gamma_{1} \kappa^{5 / 8}\right) \int_{\Omega\left(\kappa^{-1}\right)}|\psi|^{2} d x
$$

where $\mathcal{S}_{\kappa}(\mathbf{h})$ is a thin layer surrounding $(\partial \Omega)_{\mathbf{h}}$ and is defined by

$$
\mathcal{S}_{\kappa}(\mathbf{h})=\left\{x \in \Omega\left(\kappa^{-1}\right):\left|\varphi_{\mathbf{h}}(x)-\frac{\pi}{2}\right| \leq \eta \kappa^{-3 / 16}\right\} .
$$

(2) If $\sigma=\kappa / b$, where $\beta_{0}<b<1$, there exist positive constants $\vartheta(b), \eta(\kappa), \gamma_{2}$ and $M_{2}$, all depending on $b$, where $0<\vartheta(b)<\pi / 2$ and $\eta(\kappa) \rightarrow 0$ as $\kappa \rightarrow \infty$, such that for large $\kappa$,

$$
\int_{\Omega \backslash \mathcal{S}_{b, \kappa}(\mathbf{h})}|\psi|^{2} d x \leq M_{2} \exp \left(-\gamma_{2} \kappa^{3 / 4}\right) \int_{\Omega\left(\kappa^{-1}\right)}|\psi|^{2} d x,
$$

where

$$
\mathcal{S}_{b, \kappa}(\mathbf{h})=\left\{x \in \Omega\left(\kappa^{-1}\right): \vartheta(b)-\eta(\kappa) \leq \varphi_{\mathbf{h}}(x) \leq \pi-\vartheta(b)+\eta(\kappa)\right\} .
$$

\footnotetext{
${ }^{3}$ Note that, if $P_{\min }=0,(1.8)$ is still not good enough, and we should carry out further analysis to catch a non-zero next higher order term in the expansion of $H_{C_{3}}$, and we expect a formula similar to (1.1) holds, with $\kappa_{\max }$ replaced by the principal curvature corresponding to the direction orthogonal to $\mathbf{h}$.
} 
Remark 1.1. From (1.9) we see that, if the applied field $\mathcal{H}=\sigma \mathbf{h}$ is not far from $H_{C_{3}}$ in the sense that $\sigma=H_{C_{3}}(\mathbf{h}, \kappa)-a_{1} \kappa^{1 / 2}$ with $a_{1}>0$, superconductivity is confined to a vicinity of $(\partial \Omega)_{\mathbf{h}}$, and in the $L^{2}$ sense the order parameters exponentially decay away from $(\partial \Omega)_{\mathbf{h}}$ both in the direction normal to the surface and in the directions tangential to the surface.

Remark 1.2. From (1.10), if the applied field lies in-between and keeps away from $H_{C_{2}}$ and $H_{C_{3}}$ in the sense that

$$
\sigma=\frac{\kappa}{b}, \quad b \text { is fixed, } \quad \beta_{0}<b<1, \quad \kappa \text { is large, }
$$

then superconductivity is confined to the vicinity of a portion of the surface where the angle between the applied field and either the normal vector $\nu$ or $-\nu$ is smaller than $\vartheta(b)$. Note that if $\kappa$ is fixed and $b$ increases to 1 , then $\mathcal{S}_{b, \kappa}(\mathbf{h})$ expands and gradually covers the entire surface, which means that when the applied field decreases to $H_{C_{2}}$, the whole surface will turn into the superconducting state; however, before the applied field reaches $H_{C_{2}}$, there always exists a subset of the surface with positive area which remains close to the normal state. In particular, the subset of the surface that is orthogonal to the applied field is always close to the normal state. This phenomenon illustrates a significant difference between 2-dimensional superconductivity (on a cylinder of infinite height) and 3-dimensional superconductivity (on a 3-dimensional bounded domain). As was proved in [P1] and mentioned above that, for a 2-dimensional superconductor, as the applied field decreases from $H_{C_{3}}$, a superconducting sheath forms quickly over the entire boundary, and the sample turns into the surface superconducting state much earlier than the interior nucleation occurs. However, for a 3-dimensional superconductor, superconducting sheath will not cover the entire surface when the applied field is between $H_{C_{2}}$ and $H_{C_{3}}$. Thus the uniform surface superconducting state will not occur on a 3-dimensional bounded superconductor. Our Theorem 2 is a mathematical verification for the observations of Saint-James and Sarma [SST] mentioned above.

In order to estimate the value of $H_{C_{3}}$ and prove Theorem 1, we must study the eigenvalue problem for the Schrödinger operator $-\nabla_{\varepsilon^{-2} \mathbf{F}_{\mathrm{h}}}^{2}$ in $\Omega$ :

$$
-\nabla_{\varepsilon^{-2} \mathbf{F}_{\mathbf{h}}}^{2} \phi=\mu \phi \quad \text { in } \Omega, \quad\left(\nabla_{\varepsilon^{-2} \mathbf{F}_{\mathbf{h}}} \phi\right) \cdot \nu=0 \quad \text { on } \partial \Omega,
$$

where $\varepsilon>0$ is a small real number. In has been proved in [LP4] (Appendix) that, for a 3-dimensional bounded, smooth and simply-connected domain,

$$
\frac{1}{\varepsilon^{2}}\left(\beta_{0}+o(1)\right) \leq \mu\left(\varepsilon^{-2} \mathbf{F}_{\mathbf{h}}\right) \leq \frac{1}{\varepsilon^{2}}\left(\beta_{0}+C \varepsilon^{2 / 3}\right) .
$$

In this paper we are able to improve the estimate (1.13) and prove a satisfactory upper bound

$$
\mu\left(\varepsilon^{-2} \mathbf{F}_{\mathbf{h}}\right) \leq \frac{1}{\varepsilon^{2}}\left\{\beta_{0}+C_{2} P_{\min } \varepsilon^{2 / 3}+O\left(\varepsilon^{5 / 6}\right)\right\}
$$

for all small $\varepsilon>0$, where $C_{2}$ is the number given in (1.6). We expect that the equality in (1.14) holds up to an error $O\left(\varepsilon^{7 / 9}\right)$, namely

$$
\mu\left(\varepsilon^{-2} \mathbf{F}_{\mathbf{h}}\right)=\frac{1}{\varepsilon^{2}}\left\{\beta_{0}+C_{2} P_{\min } \varepsilon^{2 / 3}+O\left(\varepsilon^{7 / 9}\right)\right\} .
$$


Remark 1.3. In this paper we consider the homogeneous boundary condition for order parameters $\psi$. As in [LP1], [LP2], [LP3] and [LP4], we can also consider a boundary condition

$$
\nabla_{\kappa \sigma \mathbf{A}} \psi \cdot \nu+\gamma \psi=0
$$

with $\gamma>0$, which was posed by de Gennes dG] for superconductors adjacent to other material. It will produce more technical complexity.

This paper is organized as follows. In Section 2 we discuss several auxiliary linear differential equations, leaving some computations in Appendix A. An analysis on the local coordinates at boundary is given in Section 3 and some computational details are given in Appendix B. This analysis is needed in Section 4, where we prove (1.14) and obtain a lower bound estimate for $H_{C_{3}}$. The proof of (1.14) consists of careful analysis of functions and vector fields near a boundary point, carefully choosing test functions, and controlling error terms. In order to show clearly the intuition of choosing the test functions, we present a formal analysis in Appendix C. In Section 5 we establish the upper bound estimate for $H_{C_{3}}$ based on a series of elliptic estimates. In Section 6 we discuss the location of nucleation of superconductivity as well as the behavior of order parameters for applied fields lying in-between and keeping away from $H_{C_{2}}$ and $H_{C_{3}}$.

We proved the upper bound estimate (1.14) and conjectured the formula (1.15) independently and simultaneously with B. Helffer and A. Morame. Just after we submitted the first version of this paper in October 2001, we received from Professor Helffer his preprint with A. Morame: "Magnetic bottles for the Neumann problem: Curvature effects in the case of dimension 3", where they proved an asymptotic estimate for $\mu\left(\varepsilon^{-2} \mathbf{F}_{\mathbf{h}}\right)$ under some geometric assumptions on the domain $\Omega$. In March 2002 we received from Professor Helffer the preprint: "Magnetic bottles for the Neumann problem: Curvature effects in the case of dimension 3 (general case)", which is an improved version of the first one, and in which they proved the asymptotic estimate for $\mu\left(\varepsilon^{-2} \mathbf{F}_{\mathbf{h}}\right)$ under a very slight condition on the domain, and they also proved that their formula is equivalent to (1.15) up to the error term $O\left(\varepsilon^{2 / 3+\delta}\right)$ for some $\delta>0$. In February 2003 we received from Professor Y. Almog his preprint: "Non-linear surface superconductivity in three dimensions in the large $\kappa$ limit", where he considered superconductivity on a 3-dimensional bounded domain under an applied field $\mathcal{H}=\sigma \mathbf{h}$ with $\sigma-\kappa \gg \kappa^{1 / 2}$, and proved that order parameters exponentially decay in the normal direction away from surface.

\section{§2. Auxiliary equations}

In this section we study several auxiliary linear differential equations and collect some facts which will be used in this paper. For fixed $z$, let $\beta(z)$ denote the lowest eigenvalue of the following eigenvalue problem

$$
\left\{\begin{array}{l}
-u^{\prime \prime}+(z+t)^{2} u=\beta(z) u \quad \text { for } t>0, \\
u^{\prime}(0)=0, \quad u(+\infty)=0
\end{array}\right.
$$

The following results were proved by Dauge-Helffer in [DH]; also see [LP1], and [LP3] (Section 2 and Appendix).

Lemma 2.1. (1) There is a unique $z_{0}, z_{0}<0$, such that $\beta\left(z_{0}\right)=\inf _{z \in \mathbb{R}} \beta(z)=\beta_{0}$. Moreover, $\beta_{0}=\left|z_{0}\right|^{2}$. 
(2) Let $u(t)$ be a positive eigenfunction of (2.1) for $z=z_{0}$ associated with the lowest eigenvalue $\beta_{0} . u(t)$ is strictly decreasing in $t$ and exponentially decays as $t \rightarrow+\infty$, and

$$
\int_{0}^{+\infty}\left(z_{0}+t\right) u^{2}(t) d t=0, \quad \int_{0}^{+\infty}\left(t+z_{0}\right)^{2} u(t) d t=\frac{\beta_{0}}{2} \int_{0}^{+\infty} u^{2}(t) d t .
$$

It has been proved in [LP3] that $0.5<\beta_{0}<0.76$. The number $C_{1}$ appearing in (1.1) for 2-dimensional superconductors is defined by an eigenfunction $u$ of (2.1):

$$
C_{1}=\frac{u^{2}(0)}{3\|u\|_{L^{2}\left(\mathbb{R}_{+}\right)}^{2}} .
$$

It was shown in LP3] that

$$
C_{1}=\frac{1}{\|u\|_{L^{2}\left(\mathbb{R}_{+}\right)}^{2}}\left\{\frac{u^{2}(0)}{2}-\int_{0}^{+\infty}\left(t+z_{0}\right)^{3} u^{2}(t) d t\right\} .
$$

Lemma 2.2. Given a solution u of (2.1), the solutions of the equation

$$
\left\{\begin{array}{l}
-u_{1}^{\prime \prime}+\left(t+z_{0}\right)^{2} u_{1}-\beta_{0} u_{1}=-\left(t+z_{0}\right) u \quad \text { for } t>0, \\
u_{1}^{\prime}(0)=0, \quad u_{1}(\infty)=0
\end{array}\right.
$$

are given by $u_{1}=\frac{u^{\prime}}{2}+c u$, where $c$ is an arbitrary constant. For any solution $u_{1}$ of (2.3), let

$$
\alpha_{0}=-\frac{4}{\|u\|_{L^{2}\left(\mathbb{R}_{+}\right)}^{2}} \int_{0}^{+\infty}\left(t+z_{0}\right) u u_{1} d t .
$$

$\alpha_{0}$ is independent of the choice of $u_{1}$, and $0<\alpha_{0}<1$.

Proof. $u^{\prime \prime}(0)=0$ since $\beta_{0}=z_{0}^{2}$. Hence $\frac{1}{2} u^{\prime}(t)$ satisfies (2.3). Obviously, the solutions $u_{1}$ of (2.3) are given by $u^{\prime} / 2+c u$. Integrating by parts we find that

$$
\alpha_{0}=-\frac{4}{\|u\|_{L^{2}\left(\mathbb{R}_{+}\right)}^{2}} \int_{0}^{+\infty}\left(t+z_{0}\right) u u_{1} d t=1+\frac{z_{0} u^{2}(0)}{\|u\|_{L^{2}\left(\mathbb{R}_{+}\right)}^{2}}=1-3 C_{1}\left|z_{0}\right|<1 .
$$

On the other hand, multiplying (2.3) by $u_{1}$ and integrating, and using the simplicity of the eigenvalue $\beta_{0}$, we find that

$$
-\int_{0}^{+\infty}\left(t+z_{0}\right) u u_{1} d t=\int_{0}^{+\infty}\left\{\left|u_{1}^{\prime}\right|^{2}+\left(t+z_{0}\right)^{2} u_{1}^{2}-\beta_{0} u_{1}^{2}\right\} d t>0
$$

so $\alpha_{0}>0$.

In the following we shall always choose the positive eigenfunction $u$ of (2.1) such that $\|u\|_{L^{2}\left(\mathbb{R}_{+}\right)}=1$. Let $u_{1}=\frac{u^{\prime}}{2}+\frac{3 C_{1}}{2} u$. Then $u_{1}$ is a solution of $(2.3)$ and satisfies $\int_{0}^{+\infty} u u_{1} d t=0 . \alpha_{0}$ always denotes the number given in (2.4). $u$ and $u_{1}$ will be used to construct test functions in Sections 3 and 4.

Next, given a unit vector $\mathbf{h}$, let $\mathbf{F}_{\mathbf{h}}$ be the vector field satisfying (1.3), and let $\lambda_{\mathbf{h}}$ be the lowest eigenvalue of the following problem in the half space $\mathbb{R}_{+}^{3}=$ $\left\{\left(x_{1}, x_{2}, x_{3}\right): x_{3}>0\right\}$ :

$$
-\nabla_{\mathbf{F}_{\mathbf{h}}}^{2} \psi=\lambda \psi \quad \text { in } \mathbb{R}_{+}^{3}, \quad \nabla_{\mathbf{F}_{\mathbf{h}}} \psi \cdot \nu=0 \quad \text { on } \partial \mathbb{R}_{+}^{3} .
$$

Let $\vartheta\left(\partial \mathbb{R}_{+}^{3}, \mathbf{h}\right)$ be the angle between $\mathbf{h}$ and the unit outer normal of $\partial \mathbb{R}_{+}^{3}$. $0 \leq$ $\vartheta\left(\partial \mathbb{R}_{+}^{3}, \mathbf{h}\right) \leq \pi$. 
Lemma 2.3. There exists a continuous function $\lambda(\vartheta)$ on $[0, \pi]$ such that $\lambda_{\mathbf{h}}=$ $\lambda\left(\vartheta\left(\partial \mathbb{R}_{+}^{3}, \mathbf{h}\right)\right)$ for all unit vectors $\mathbf{h}$.

(1) $\lambda(\vartheta)$ is strictly decreasing for $0 \leq \vartheta \leq \pi / 2, \lambda(0)=1, \lambda\left(\frac{\pi}{2}\right)=\beta_{0}$, and $\lambda(\vartheta)=\lambda(\pi-\vartheta)$.

(2) For $0<\vartheta<\pi / 2$, it holds that

$$
\beta_{0} \sin ^{2} \vartheta+\cos ^{2} \vartheta<\lambda(\vartheta)<\beta_{0} \sin \vartheta+\cos \vartheta .
$$

(3) For small $\varepsilon>0$,

$$
\begin{array}{ll}
\left|\vartheta-\frac{\pi}{2}\right| \leq\left(1-\beta_{0}\right)^{-1 / 2} \varepsilon^{1 / 2}+O\left(\varepsilon^{3 / 2}\right) & \text { if } \lambda(\vartheta) \leq \beta_{0}+\varepsilon, \\
\left|\vartheta-\frac{\pi}{2}\right| \geq \varepsilon+O\left(\varepsilon^{2}\right) & \text { if } \lambda(\vartheta) \geq \beta_{0}+\varepsilon .
\end{array}
$$

Proof. The conclusions of Lemma 2.3, except (2) and (3), were established in [LP4] (Theorem 4.2). It was also proved there that, if $0<\vartheta<\pi / 2$, then $\lambda(\vartheta)=b(\vartheta)$, where

$$
b(\vartheta)=\inf _{\phi \in W^{1,2}\left(\mathbb{R}_{+}^{2}\right)} \frac{\int_{\mathbb{R}_{+}^{2}}\left\{|\nabla \phi|^{2}+\left(x_{1} \cos \vartheta-x_{2} \sin \vartheta\right)^{2}|\phi|^{2}\right\} d x}{\|\phi\|_{L^{2}\left(\mathbb{R}_{+}^{2}\right)}^{2}} .
$$

The right side inequality of (2.5) was proved in [LP4] (Lemma 3.8) by making a change of variables. To prove the left side inequality, we make a change of variables $y_{1}=x_{1} \cos \vartheta, y_{2}=x_{2} \sin \vartheta, w(y)=\phi(x)$, and find

$$
\begin{aligned}
b(\vartheta)= & \inf _{w \in W^{1,2}\left(\mathbb{R}_{+}^{2}\right)}\left\{\cos ^{2} \vartheta \frac{\int_{\mathbb{R}_{+}^{2}}\left[\left|\partial_{y_{1}} w\right|^{2}+\left(y_{1}-y_{2}\right)^{2}|w|^{2}\right] d y}{\|w\|_{L^{2}\left(\mathbb{R}_{+}^{2}\right)}^{2}}\right. \\
& \left.+\sin ^{2} \vartheta \frac{\int_{\mathbb{R}_{+}^{2}}\left[\left|\partial_{y_{2}} w\right|^{2}+\left(y_{2}-y_{1}\right)^{2}|w|^{2}\right] d y}{\|w\|_{L^{2}\left(\mathbb{R}_{+}^{2}\right)}^{2}}\right\} \\
\geq & \cos ^{2} \vartheta \inf _{w \in W^{1,2}\left(\mathbb{R}_{+}^{2}\right)} \frac{\int_{\mathbb{R}_{+}^{2}}\left[\left|\partial_{y_{1}} w\right|^{2}+\left(y_{1}-y_{2}\right)^{2}|w|^{2}\right] d y}{\|w\|_{L^{2}\left(\mathbb{R}_{+}^{2}\right)}^{2}} \\
+ & \sin ^{2} \vartheta \inf _{w \in W^{1,2}\left(\mathbb{R}_{+}^{2}\right)} \frac{\int_{\mathbb{R}_{+}^{2}}\left[\left|\partial_{y_{2}} w\right|^{2}+\left(y_{2}-y_{1}\right)^{2}|w|^{2}\right] d y}{\|w\|_{L^{2}\left(\mathbb{R}_{+}^{2}\right)}^{2}}=\cos ^{2} \vartheta+\beta_{0} \sin ^{2} \vartheta .
\end{aligned}
$$

Therefore, $b(\vartheta) \geq \cos ^{2} \vartheta+\beta_{0} \sin ^{2} \vartheta$. If the equality holds for some $0<\vartheta<\pi / 2$, then $b(\vartheta)$ is achieved by a function $\phi(x) \in W^{1,2}\left(\mathbb{R}_{+}^{2}\right)$ (see [LP4], Theorem 3.1). Let

$$
w(y)=\phi\left(\frac{y_{1}}{\cos \vartheta}, \frac{y_{2}}{\sin \vartheta}\right) .
$$

Then $w(y)$ must satisfy

$$
\int_{\mathbb{R}_{+}^{2}}\left[\left|\partial_{y_{2}} w\right|^{2}+\left(y_{2}-y_{1}\right)^{2}|w|^{2}\right] d y=\beta_{0}\|w\|_{L^{2}\left(\mathbb{R}_{+}^{2}\right)}^{2} .
$$

This contradicts conclusion (1) of Lemma 2.1.

To prove (3), we assume $0<\vartheta<\pi / 2$. If $\lambda(\vartheta) \leq \beta_{0}+\varepsilon$, from (2.5) we have

$$
\beta_{0} \sin ^{2} \vartheta+\cos ^{2} \vartheta \leq \lambda(\vartheta) \leq \beta_{0}+\varepsilon,
$$

\footnotetext{
${ }^{4}$ Such techniques were used several times in [LP4] and [P2] in the estimates of various eigenvalue problems.
} 
so $\cos \vartheta \leq \sqrt{\varepsilon /\left(1-\beta_{0}\right)}$, and hence

$$
\frac{\pi}{2}-\vartheta \leq \sqrt{\frac{\varepsilon}{1-\beta_{0}}}+O\left(\varepsilon^{3 / 2}\right) .
$$

If $\lambda(\vartheta) \geq \beta_{0}+\varepsilon$, using (2.5) again we have

$$
\beta_{0} \sin \vartheta+\cos \vartheta \geq \lambda(\vartheta) \geq \beta_{0}+\varepsilon,
$$

and hence $f(\vartheta) \equiv \beta_{0}(\sin \vartheta-1)+\cos \vartheta \geq \varepsilon$. The function $f(\vartheta)$ is strictly decreasing for $\vartheta \in\left(\arctan \left(\beta_{0}\right), \pi / 2\right)$. So $\vartheta \leq f^{-1}(\varepsilon) \equiv \vartheta_{\varepsilon}$. Let $t_{\varepsilon}=\pi / 2-\vartheta_{\varepsilon}$. Then

$$
\varepsilon=f\left(\vartheta_{\varepsilon}\right)=f\left(\frac{\pi}{2}-t_{\varepsilon}\right)=\beta_{0}\left(\cos t_{\varepsilon}-1\right)+\sin t_{\varepsilon}=t_{\varepsilon}-\frac{\beta_{0}}{2} t_{\varepsilon}^{2}+O\left(t_{\varepsilon}^{3}\right) .
$$

Thus $t_{\varepsilon}=O(\varepsilon)$ and $\frac{\beta_{0}}{2} t_{\varepsilon}^{2}-t_{\varepsilon}+\varepsilon+O\left(\varepsilon^{3}\right)=0$. So

$$
t_{\varepsilon}=\frac{1}{\beta_{0}}\left[1-\sqrt{1-2 \beta_{0} \varepsilon+O\left(\varepsilon^{3}\right)}\right]=\varepsilon+O\left(\varepsilon^{2}\right),
$$

and

$$
\frac{\pi}{2}-\vartheta \geq \frac{\pi}{2}-\vartheta_{\varepsilon}=t_{\varepsilon}=\varepsilon+O\left(\varepsilon^{2}\right) .
$$

We would like to mention that the inequality $\lambda(\vartheta) \geq \cos ^{2} \vartheta+\beta_{0} \sin ^{2} \vartheta$ for $0<$ $\vartheta<\pi / 2$ has been proved in [HM2]. Our argument yields the strict inequality.

Remark 2.4. Let $\varphi_{\mathbf{h}}(x)$ be the angle between $\mathbf{h}$ and the outer normal vector $\nu(x)$ on $\partial \Omega$, as defined in Section 1. $\varphi_{\mathbf{h}}(x)$ is well defined on $\partial \Omega$ and is continuously differentiable in the set $\left\{x \in \partial \Omega: 0<\varphi_{\mathbf{h}}(x)<\pi\right\}$. Let $\lambda(\vartheta)$ be the function given in Lemma 2.3. Then $\lambda\left(\varphi_{\mathbf{h}}(x)\right)$ is a continuous function, and

$$
\lambda\left(\varphi_{\mathbf{h}}(x)\right)=\beta_{0} \quad \text { on }(\partial \Omega)_{\mathbf{h}}, \quad \beta_{0}<\lambda\left(\varphi_{\mathbf{h}}(x)\right) \leq 1 \quad \text { on } \partial \Omega \backslash(\partial \Omega)_{\mathbf{h}} .
$$

In Sections 5 and 6 we shall extend the function $\varphi_{\mathbf{h}}(x)$ onto $\bar{\Omega}$. The extension can be done as follows. There exists a number $\delta_{0}>0$ such that, for any $x \in \Omega\left(\delta_{0}\right)$ we can find a unique point $P(x) \in \partial \Omega$ such that $|P(x)-x|=\operatorname{dist}(x, \partial \Omega)$. Then we define

$$
\varphi_{\mathbf{h}}(x)=\varphi_{\mathbf{h}}(P(x)) \quad \text { for } x \in \Omega\left(\delta_{0}\right) .
$$

We can further extend it over $\bar{\Omega}$ to get a continuous function on $\bar{\Omega}$, such that $0 \leq \varphi_{\mathbf{h}}(x) \leq \pi$, and $\varphi_{\mathbf{h}}(x)$ is continuously differentiable in the set $\left\{x \in \Omega\left(\delta_{0}\right): 0<\right.$ $\left.\varphi_{\mathbf{h}}(x)<\pi\right\}$. Now $\lambda\left(\varphi_{\mathbf{h}}(x)\right)$ is also well defined and continuous on $\bar{\Omega}$, and $\beta_{0} \leq$ $\lambda\left(\varphi_{\mathbf{h}}(x)\right) \leq 1$.

In Section 3 we need bounded solutions of an Euler equation associated with the variational problem for the functional $p$ :

$$
\begin{aligned}
p[\phi]=\int_{\mathbb{R}^{2}}\left\{c_{11}\left|\partial_{y_{1}} \phi+i a_{2}^{(1)} y_{2}^{2} \phi\right|^{2}+2 c_{12} \Re\left[\left(\partial_{y_{1}} \phi\right.\right.\right. & \left.\left.+i a_{2}^{(1)} y_{2}^{2} \phi\right)\left(\partial_{y_{2}} \bar{\phi}+i a_{1}^{(2)} y_{1}^{2} \bar{\phi}\right)\right] \\
& \left.+c_{22}\left|\partial_{y_{2}} \phi-i a_{1}^{(2)} y_{1}^{2} \phi\right|^{2}\right\} d y
\end{aligned}
$$

Here $a_{2}^{(1)}, a_{1}^{(2)}$ are real numbers, $c_{11}>0, c_{22}>0$, and $c_{11} c_{22}-c_{12}^{2}>0$. Define

$$
\mu_{p}=\inf _{\phi \in \mathcal{W}\left(\mathbb{R}^{2}\right)} \frac{p[\phi]}{\|\phi\|_{L^{2}\left(\mathbb{R}^{2}\right)}^{2}}
$$


where

$$
\mathcal{W}\left(\mathbb{R}^{2}\right)=\left\{\phi \in L^{2}\left(\mathbb{R}^{2}\right) \cap W_{\mathrm{loc}}^{1,2}\left(\mathbb{R}^{2}\right): p[\phi]<\infty\right\} .
$$

We can show that the infimum $\mu_{p}$ is not achieved in $\mathcal{W}\left(\mathbb{R}^{2}\right)$, however, the associated Euler equation

$$
\begin{aligned}
c_{11}\left(\partial_{y_{1}}+\right. & \left.i a_{2}^{(1)} y_{2}^{2}\right)^{2} \psi+c_{12}\left\{\left(\partial_{y_{1}}+i a_{2}^{(1)} y_{2}^{2}\right)\left(\partial_{y_{2}}-i a_{1}^{(2)} y_{1}^{2}\right) \psi\right. \\
& \left.+\left(\partial_{y_{2}}-i a_{1}^{(2)} y_{1}^{2}\right)\left(\partial_{y_{1}}+i a_{2}^{(1)} y_{2}^{2}\right) \psi\right\}+c_{22}\left(\partial_{y_{2}}-i a_{1}^{(2)} y_{1}^{2}\right)^{2} \psi+\mu_{p} \psi=0
\end{aligned}
$$

in $\mathbb{R}^{2}$ has a nontrivial bounded solution $\psi(y)$. This equation can be written as follows:

$$
c_{11} \partial_{y_{1} y_{1}} \psi+2 c_{12} \partial_{y_{1} y_{2}} \psi+c_{22} \partial_{y_{2} y_{2}} \psi-2 i f_{1} \partial_{y_{1}} \psi-2 i f_{2} \partial_{y_{2}} \psi-f_{0} \psi+\mu_{p} \psi=0
$$

where

$$
\begin{aligned}
f_{0}= & c_{11}\left(a_{2}^{(1)}\right)^{2} y_{2}^{4}-2 c_{12} a_{2}^{(1)} a_{1}^{(2)} y_{1}^{2} y_{2}^{2}+c_{22}\left(a_{1}^{(2)}\right)^{2} y_{1}^{4} \\
& +2 i c_{12}\left(a_{1}^{(2)} y_{1}-a_{2}^{(1)} y_{2}\right) \\
f_{1}= & c_{12} a_{1}^{(2)} y_{1}^{2}-c_{11} a_{2}^{(1)} y_{2}^{2} \\
f_{2}= & c_{22} a_{1}^{(2)} y_{1}^{2}-c_{12} a_{2}^{(1)} y_{2}^{2} .
\end{aligned}
$$

In order to simplify equation (2.8), we introduce new variables $\xi=\left(\xi_{1}, \xi_{2}\right)$ by

$$
\begin{aligned}
& y_{1}=\frac{\sqrt{c_{11}}}{2}\left(\varepsilon_{1} \xi_{1}+\varepsilon_{2} \xi_{2}\right), \\
& y_{2}=\frac{\sqrt{c_{22}}}{2}\left(\varepsilon_{1} \xi_{1}-\varepsilon_{2} \xi_{2}\right), \\
& \psi(y)=\psi_{1}(\xi)
\end{aligned}
$$

where

$$
\varepsilon_{1}=\left(2+\frac{2 c_{12}}{\sqrt{c_{11} c_{22}}}\right)^{1 / 2}, \quad \varepsilon_{2}=\left(2-\frac{2 c_{12}}{\sqrt{c_{11} c_{22}}}\right)^{1 / 2} .
$$

Then we can write the equation for $\psi_{1}(\xi)$ in the following form:

$$
-\nabla_{\mathbf{Q}}^{2} \psi_{1}=\mu_{2} \psi_{1} \quad \text { in } \mathbb{R}^{2},
$$

where

$$
\mathbf{Q}(\xi)=-\frac{|\xi|^{2}}{2}\left(\gamma_{1}, \gamma_{2}\right)+\nabla \eta(\xi)
$$

Here $\eta(\xi)$ is a homogeneous polynomial of $\xi_{1}, \xi_{2}$ of degree 3 , and

$$
\begin{aligned}
& \gamma_{1}=2 \frac{c_{11} c_{22}-\left(c_{12}\right)^{2}}{\varepsilon_{1} \sqrt{c_{11} c_{22}}}\left(\sqrt{c_{22}} a_{2}^{(1)}-\sqrt{c_{11}} a_{1}^{(2)}\right), \\
& \gamma_{2}=2 \frac{c_{11} c_{22}-\left(c_{12}\right)^{2}}{\varepsilon_{2} \sqrt{c_{11} c_{22}}}\left(\sqrt{c_{22}} a_{2}^{(1)}+\sqrt{c_{11}} a_{1}^{(2)}\right) .
\end{aligned}
$$

For the details see Appendix A.

Let us introduce $\rho$ and $\vartheta$ by

$$
\rho=\left(\gamma_{1}^{2}+\gamma_{2}^{2}\right)^{1 / 2}, \quad \gamma_{1}=\rho \cos \vartheta, \quad \gamma_{2}=\rho \sin \vartheta
$$


and introduce new variables $x=\left(x_{1}, x_{2}\right)$ by

$$
\begin{aligned}
& \xi_{1}=\frac{1}{\rho^{1 / 3}}\left(x_{1} \cos \vartheta-x_{2} \sin \vartheta\right), \\
& \xi_{2}=\frac{1}{\rho^{1 / 3}}\left(x_{1} \sin \vartheta+x_{2} \cos \vartheta\right), \\
& \varphi(x)=\psi_{1}(\xi) \exp \left(\frac{i}{6} x_{1}^{3}-i \eta(\xi)\right) .
\end{aligned}
$$

Let

$$
\lambda_{0}=\mu_{p} \rho^{-2 / 3} \text {. }
$$

Then we can write the equation for $\varphi(x)$ as follows:

$$
-\Delta \varphi-i x_{2}^{2} \partial_{x_{1}} \varphi+\frac{1}{4} x_{2}^{4} \varphi=\lambda_{0} \varphi \quad \text { in } \mathbb{R}^{2} .
$$

Equation (2.14) has been studied by Montgomery [M], Helffer [H] and Pan-Kwek PK]. Montgomery [M] proved that the lowest eigenvalue $\lambda_{0}$ of $(2.14)$ is given by $\lambda_{0}=\inf _{\tau \in \mathbb{R}} \lambda(\tau)$, where $\lambda(\tau)$ is the lowest eigenvalue of the following ordinary differential equation

$$
-v^{\prime \prime}+\frac{1}{4}\left(t^{2}+2 \tau\right)^{2} v=\lambda(\tau) v, \quad-\infty<t<\infty .
$$

Pan-Kwek PK] further showed that the function $\lambda(\tau)$ has a unique minimum point $\tau_{0}, \tau_{0}<0$,

$$
\lambda\left(\tau_{0}\right)=\lambda_{0}=\inf _{\tau \in \mathbb{R}} \lambda(\tau),
$$

and the eigenfunctions of (2.14) corresponding to $\lambda_{0}$ are given by

$$
\varphi(x)=c e^{i \tau_{0} x_{1}} v\left(x_{2}\right),
$$

where $v(t)$ is the eigenfunction of (2.15) with $\tau=\tau_{0}$. Hence, the lowest eigenvalue of (2.8) is $\mu_{p}=\lambda_{0} \rho^{2 / 3}$, and the bounded eigenfunctions do not lie in $\mathcal{W}\left(\mathbb{R}^{2}\right)$. Thus we have obtained the following conclusion.

Proposition 2.5. The lowest eigenvalue of (2.8) is

$$
\mu_{p}=\lambda_{0}\left(\gamma_{1}^{2}+\gamma_{2}^{2}\right)^{1 / 3}
$$

where $\gamma_{1}, \gamma_{2}$ are given in (2.12), and $\lambda_{0}$ is the lowest eigenvalue of (2.14). $\mu_{p}$ is not achieved in $\mathcal{W}\left(\mathbb{R}^{2}\right)$. After the transformations of coordinates (2.10) and (2.13), the bounded solutions of (2.8) associated with the lowest eigenvalue $\mu_{p}$ are $\psi(y)=c \psi_{p}(y)$, where

$$
\psi_{p}(y)=v\left(x_{2}\right) \exp \left(i \tau_{0} x_{1}-\frac{i}{6} x_{1}^{3}+i \eta(\xi)\right) ;
$$

here $\tau_{0}$ is given in (2.16), $v$ is the eigenfunction of (2.15) with $\tau=\tau_{0}$, and $\eta(\xi)$ is a homogeneous polynomial of degree 3 .

Remark 2.6. Let $\psi_{p}(y)$ be the function given in (2.18). For any $R_{1}, R_{2}>0$, we have

$$
\int_{\left\{\left|y_{1}\right|<R_{1},\left|y_{2}\right|<R_{2}\right\}} y_{j}\left|\psi_{p}(y)\right|^{2} d y=0, \quad j=1,2 .
$$




\section{§3. LOCAL COORDINATES NEAR BOUNDARY}

In this section we give some analysis in the local coordinates determined by a diffeomorphism that straightens a piece of surface. This analysis is needed in Section 4 to obtain estimates of the eigenvalues. Let us fix a point $X_{0} \in \partial \Omega$, and introduce new variables $y_{1}, y_{2}$ such that $\partial \Omega$ be represented (at least near $X_{0}$ ) by $\mathbf{r}=\mathbf{r}\left(y_{1}, y_{2}\right)$, and $\mathbf{r}(0,0)=X_{0}$. Here and henceforth we let

$$
y=\left(y_{1}, y_{2}\right)
$$

and use the notation $\mathbf{r}_{j}(y)=\partial_{y_{j}} \mathbf{r}(y), \mathbf{r}_{i j}=\partial_{y_{i} y_{j}} \mathbf{r}(y)$, etc. Let

$$
\mathbf{n}(y)=\frac{\mathbf{r}_{1}(y) \times \mathbf{r}_{2}(y)}{\left|\mathbf{r}_{1}(y) \times \mathbf{r}_{2}(y)\right|} \text {. }
$$

We choose $\left(y_{1}, y_{2}\right)$ in such a way that $\mathbf{n}(y)$ is the inward normal of $\partial \Omega$; and that the $y_{1}$ - and $y_{2}$-curves on $\partial \Omega$ are the lines of curvatures; thus, $\mathbf{r}_{1}(y)$ and $\mathbf{r}_{2}(y)$ are orthogonal to each other everywhere on $\partial \Omega$. Let

$$
g_{i j}(y)=\mathbf{r}_{i}(y) \cdot \mathbf{r}_{j}(y), \quad g(y)=\operatorname{det}\left(g_{i j}(y)\right)=g_{11}(y) g_{22}(y) .
$$

Let $\Omega_{i j}(y)$ denote the coefficients of the second fundamental form of $\partial \Omega$. According to the choice of $y_{1}$ and $y_{2}$, we have

$$
\Omega_{11}=g_{11} \kappa_{1}, \quad \Omega_{12}=0, \quad \Omega_{22}=g_{22} \kappa_{2} ;
$$

here $\kappa_{1}$ and $\kappa_{2}$ are the principal curvatures of $\partial \Omega$. Let $\partial_{j}$ denote $\partial_{y_{j}}$ for $j=1,2$, and $\partial_{3}$ denote $\partial_{z}$. For scalar functions $f$, let $f_{, j}$ denote the partial derivative in $y_{j}$. Let us define a map $\mathcal{F}$ by

$$
X=\mathcal{F}(y, z)=\mathbf{r}\left(y_{1}, y_{2}\right)+z \mathbf{n}\left(y_{1}, y_{2}\right) .
$$

$\mathcal{F}$ is a diffeomorphism in a small neighborhood of $O$. As in [LP4 (Appendix) we calculate $\partial_{j} \mathcal{F}$ and $G_{i j}(y, z)=\partial_{i} \mathcal{F} \cdot \partial_{j} \mathcal{F}$ :

$$
\begin{aligned}
\partial_{1} \mathcal{F}=\left[1-\kappa_{1}(y) z\right] \mathbf{r}_{1}(y), \quad \partial_{2} \mathcal{F}=\left[1-\kappa_{2}(y) z\right] \mathbf{r}_{2}(y), \quad \partial_{z} \mathcal{F}=\mathbf{n}(y) ; \\
G_{11}(y, z)=g_{11}(y)\left[1-\kappa_{1}(y) z\right]^{2}, \\
G_{22}(y, z)=g_{22}(y)\left[1-\kappa_{2}(y) z\right]^{2}, \\
G_{33}(y, z)=1 \\
G_{12}(y, z)=G_{13}(y, z)=G_{23}(y, z)=0, \\
G(y, z)=\operatorname{det}\left(G_{i j}(y, z)\right)=G_{11}(y, z) G_{22}(y, z) .
\end{aligned}
$$

Let $G^{i j}(y, z)$ denote the elements of the inverse of the matrix $\left(G_{i j}(y, z)\right)$. Then

$$
G^{11}=\frac{1}{G_{11}}, \quad G^{22}=\frac{1}{G_{22}}, \quad G^{33}=1, \quad G^{12}=G^{13}=G^{23}=0 .
$$

Given a function $\psi$ and a vector field $\mathbf{A}$, in the set $\Omega(\delta)$ (see Section 1 for the definition) we can write them in the new variables $(y, z)$ as follows:

$$
\begin{aligned}
& \tilde{\psi}(y, z)=\psi(\mathcal{F}(y, z)), \\
& \tilde{\mathbf{A}}(y, z)=\mathbf{A}(\mathcal{F}(y, z))=\sum_{j=1}^{3} G^{j j}(y, z) a_{j}(y, z) \partial_{j} \mathcal{F}(y, z), \\
& \text { where } \quad a_{j}(y, z)=\mathbf{A}(\mathcal{F}(y, z)) \cdot \partial_{j} \mathcal{F}(y, z) .
\end{aligned}
$$


Later we need the Taylor expansions near $X_{0}$ of various functions and vector fields. Let

$$
\mathbf{e}_{1}=\mathbf{r}_{1}(0,0), \quad \mathbf{e}_{2}=\mathbf{r}_{2}(0,0), \quad \mathbf{e}_{3}=\mathbf{n}(0,0) .
$$

Note that $\mathbf{e}_{1}$ and $\mathbf{e}_{2}$ may not be unit vectors. In the following computations, $g, g_{, j}$, $g_{j j}, \kappa_{j}, \Omega_{j j}$, etc. denote the values of these quantities at $X_{0}$. The indices $i, j, k, l$, $m$, etc. run from 1 to 2 . We also take the summation convention, that is, when the summation is taken over repeated indices, the summation symbol is omitted. We have

$$
\begin{aligned}
& \mathbf{r}_{1}(y)=\left(1+\Gamma_{1 j}^{1} y_{j}\right) \mathbf{e}_{1}+\Gamma_{1 j}^{2} y_{j} \mathbf{e}_{2}+\Omega_{11} y_{1} \mathbf{e}_{3}+O\left(|y|^{2}\right), \\
& \mathbf{r}_{2}(y)=\Gamma_{2 j}^{1} y_{j} \mathbf{e}_{1}+\left(1+\Gamma_{2 j}^{2} y_{j}\right) \mathbf{e}_{2}+\Omega_{22} y_{2} \mathbf{e}_{3}+O\left(|y|^{2}\right), \\
& \mathbf{n}(y)=\mathbf{e}_{3}-g^{j j} \Omega_{j j} y_{j} \mathbf{e}_{j}+O\left(|y|^{2}\right)
\end{aligned}
$$

and

$$
\begin{aligned}
g_{11}(y) & =g_{11}+g_{11, j} y_{j}+O\left(|y|^{2}\right), \\
g_{22}(y) & =g_{22}+g_{22, j} y_{j}+O\left(|y|^{2}\right), \\
\sqrt{g(y)} & =\sqrt{g}\left[1+\frac{1}{2}\left(\frac{g_{11, j}}{g_{11}}+\frac{g_{22, j}}{g_{22}}\right) y_{j}\right]+O\left(|y|^{2}\right), \\
\sqrt{\frac{g_{11}(y)}{g_{22}(y)}} & =\sqrt{\frac{g_{11}}{g_{22}}}\left[1+\frac{1}{2}\left(\frac{g_{11, j}}{g_{11}}-\frac{g_{22, j}}{g_{22}}\right) y_{j}\right]+O\left(|y|^{2}\right), \\
\sqrt{\frac{g_{22}(y)}{g_{11}(y)}} & =\sqrt{\frac{g_{22}}{g_{11}}}\left[1+\frac{1}{2}\left(\frac{g_{22, j}}{g_{22}}-\frac{g_{11, j}}{g_{11}}\right) y_{j}\right]+O\left(|y|^{2}\right),
\end{aligned}
$$

where $\Gamma_{i j}^{k}$ denote the value of the Christoffel symbols at $X_{0}$, and $|y|=\sqrt{y_{1}^{2}+y_{2}^{2}}$. Let $O_{k}$ denote $O\left(|y|^{k}+|z|^{k}\right)$. We have

$$
\begin{aligned}
& G_{11}(y, z)=g_{11}+g_{11, j} y_{j}-2 \Omega_{11} z+O_{2}, \\
& G_{22}(y, z)=g_{22}+g_{22, j} y_{j}-2 \Omega_{22} z+O_{2}, \\
& G(y, z)=g+g_{, j} y_{j}-2 g\left(\kappa_{1}+\kappa_{2}\right) z+O_{2}, \\
& \sqrt{G(y, z)}=\sqrt{g}\left[1+\frac{g_{, j} y_{j}}{2 g}-\left(\kappa_{1}+\kappa_{2}\right) z\right]+O_{2}, \\
& \sqrt{\frac{G_{22}(y, z)}{G_{11}(y, z)}}=\sqrt{\frac{g_{22}}{g_{11}}}\left[1+\frac{1}{2}\left(\frac{g_{22, j}}{g_{22}}-\frac{g_{11, j}}{g_{11}}\right) y_{j}+\left(\kappa_{1}-\kappa_{2}\right) z\right]+O_{2}, \\
& \sqrt{\frac{G_{11}(y, z)}{G_{22}(y, z)}}=\sqrt{\frac{g_{11}}{g_{22}}}\left[1+\frac{1}{2}\left(\frac{g_{11, j}}{g_{11}}-\frac{g_{22, j}}{g_{22}}\right) y_{j}+\left(\kappa_{2}-\kappa_{1}\right) z\right]+O_{2} .
\end{aligned}
$$

Now we fix a unit vector $\mathbf{h}$. Let

$$
h_{1}=\mathbf{h} \cdot \mathbf{e}_{1}, \quad h_{2}=\mathbf{h} \cdot \mathbf{e}_{2}, \quad h_{3}=\mathbf{h} \cdot \mathbf{e}_{3} .
$$

We have

$$
\frac{h_{1}^{2}}{g_{11}}+\frac{h_{2}^{2}}{g_{22}}+h_{3}^{2}=1
$$

Note that if $X_{0} \in(\partial \Omega)_{\mathbf{h}}$, then $h_{3}=0$. 
Lemma 3.1. Assume $X_{0} \in(\partial \Omega)_{\mathbf{h}}$. Given any constant $\xi_{0}$, we can define a vector field $\mathbf{F}_{\mathbf{h}}$ in a neighborhood of $X_{0}$ such that curl $\mathbf{F}_{\mathbf{h}}=\mathbf{h}$, and in the coordinates $(y, z)$ introduced above with $\mathbf{r}(0,0)=X_{0}, \mathbf{F}_{\mathbf{h}}$ has the expression

$$
\mathbf{F}_{\mathbf{h}}(y, z)=\sum_{j=1}^{3} G^{j j}(y, z) \hat{a}_{j}(y, z) \partial_{j} \mathcal{F}(y, z),
$$

where

$$
\begin{aligned}
& \begin{aligned}
\hat{a}_{1}(y, z)=h_{2} & \sqrt{\frac{g_{11}}{g_{22}}}\left(z+\xi_{0}\right)-a_{2}^{(1)} y_{2}^{2}+a_{13}^{(1)} y_{1}\left(z+\xi_{0}\right) \\
& +a_{23}^{(1)} y_{2}\left(z+\xi_{0}\right)+a_{33}^{(1)} z^{2}+O_{3},
\end{aligned} \\
& \begin{aligned}
\hat{a}_{2}(y, z)=- & h_{1} \sqrt{\frac{g_{22}}{g_{11}}}\left(z+\xi_{0}\right)+a_{1}^{(2)} y_{1}^{2}+a_{13}^{(2)} y_{1}\left(z+\xi_{0}\right) \\
& +a_{23}^{(2)} y_{2}\left(z+\xi_{0}\right)+a_{33}^{(2)} z^{2}+O_{3},
\end{aligned} \\
& \hat{a}_{3}(y, z)=0,
\end{aligned}
$$

where

$$
\begin{aligned}
& a_{2}^{(1)}=-\frac{h_{2}}{2} \sqrt{g} \kappa_{2}, \\
& a_{13}^{(1)}=\frac{1}{2 \sqrt{g}}\left(h_{1} g_{11,2}+h_{2} g_{11,1}\right), \\
& a_{23}^{(1)}=\frac{1}{2 \sqrt{g}}\left(-h_{1} g_{22,1}+h_{2} g_{11,2}\right), \\
& a_{33}^{(1)}=-\frac{h_{2}}{2} \sqrt{\frac{g_{11}}{g_{22}}} \kappa_{1}, \\
& a_{1}^{(2)}=-\frac{h_{1}}{2} \sqrt{g} \kappa_{1}, \\
& a_{13}^{(2)}=a_{23}^{(1)}, \\
& a_{23}^{(2)}=-\frac{1}{2 \sqrt{g}}\left(h_{1} g_{22,2}+h_{2} g_{22,1}\right), \\
& a_{33}^{(2)}=\frac{h_{1}}{2} \sqrt{\frac{g_{22}}{g_{11}}} \kappa_{2} .
\end{aligned}
$$

The conclusion is obtained by direct computations. The details are given in Appendix B.

Under the conditions of Lemma 3.1, the eigenvalue equation for the operator $-\nabla_{\varepsilon^{-2} \mathbf{F}_{\mathbf{h}}}^{2}$ can be written as follows:

$$
\begin{array}{r}
\sum_{j=1}^{2} \frac{1}{G_{j j}(y, z)}\left\{\partial_{j j} \psi-2 i \varepsilon^{-2} \hat{a}_{j}(y, z) \partial_{1} \psi-\varepsilon^{-4}\left|\hat{a}_{j}(y, z)\right|^{2} \psi\right\}+\partial_{33} \psi \\
+\sum_{j=1}^{3} \beta_{j}(y, z) \partial_{j} \psi-i \varepsilon^{-2} \sum_{j=1}^{2}\left\{\frac{\alpha_{j}(y, z)}{\sqrt{G(y, z)}} \partial_{j} \hat{a}_{j}(y, z)\right. \\
\left.+\beta_{j}(y, z) \hat{a}_{j}(y, z)\right\} \psi+\mu \psi=0
\end{array}
$$


where

$$
\begin{aligned}
& \alpha_{1}(y, z)=\sqrt{\frac{G_{22}(y, z)}{G_{11}(y, z)}}, \quad \alpha_{2}(y, z)=\sqrt{\frac{G_{11}(y, z)}{G_{22}(y, z)}}, \quad \alpha_{3}(y, z)=\sqrt{G(y, z)}, \\
& \beta_{j}(y, z)=\frac{\partial_{j} \alpha_{j}(y, z)}{\sqrt{G(y, z)}}, \quad j=1,2,3 .
\end{aligned}
$$

The boundary condition is

$$
\partial_{z} \psi(y, 0)=0 \quad \text { if } z=0 .
$$

For later use we choose the constant $\xi_{0}$ in Lemma 3.1 to be $\varepsilon z_{0}$, where $z_{0}$ is the number given in Lemma 2.1. Consider the following rescaling for small $\varepsilon>0$ :

$$
\begin{aligned}
& \phi(y, z)=\psi\left(\varepsilon^{2 / 3} y, \varepsilon z\right), \\
& G_{j j, \varepsilon}(y, z)=G_{j j}\left(\varepsilon^{2 / 3}, \varepsilon z\right), \\
& G_{\varepsilon}(y, z)=G\left(\varepsilon^{2 / 3} y, \varepsilon z\right), \\
& b_{j}(y, z)=\varepsilon^{-1} \hat{a}_{j}\left(\varepsilon^{2 / 3} y, \varepsilon z\right), \\
& \alpha_{j, \varepsilon}(y, z)=\alpha_{j}\left(\varepsilon^{2 / 3} y, \varepsilon z\right), \\
& \beta_{j, \varepsilon}(y, z)=\beta_{j}\left(\varepsilon^{2 / 3} y, \varepsilon z\right), \quad j=1,2,3 .
\end{aligned}
$$

From (3.9) we find that, when $X_{0} \in(\partial \Omega)_{\mathbf{h}}$,

$$
\begin{aligned}
b_{1}(y, z)=h_{2} & \sqrt{\frac{g_{11}}{g_{22}}}\left(z+z_{0}\right)-a_{2}^{(1)} \varepsilon^{1 / 3} y_{2}^{2} \\
& +\varepsilon^{2 / 3}\left[a_{13}^{(1)} y_{1}+a_{23}^{(1)} y_{2}\right]\left(z+z_{0}\right)+a_{33}^{(1)} \varepsilon z^{2} \\
& +O(\varepsilon)|y|^{3}+O\left(\varepsilon^{4 / 3}\right), \\
b_{2}(y, z)=- & h_{1} \sqrt{\frac{g_{22}}{g_{11}}}\left(z+z_{0}\right)+a_{1}^{(2)} \varepsilon^{1 / 3} y_{1}^{2} \\
& +\varepsilon^{2 / 3}\left[a_{13}^{(2)} y_{1}+a_{23}^{(2)} y_{2}\right]\left(z+z_{0}\right)+a_{33}^{(2)} \varepsilon z^{2} \\
& +O(\varepsilon)|y|^{3}+O\left(\varepsilon^{4 / 3}\right),
\end{aligned}
$$

Equation (3.11) is changed to

$$
\begin{aligned}
& \sum_{j=1}^{2} \frac{1}{G_{j j, \varepsilon}(y, z)}\left\{\varepsilon^{2 / 3} \partial_{j j} \phi-2 i \varepsilon^{1 / 3} b_{j}(y, z) \partial_{j} \phi-\left|b_{j}(y, z)\right|^{2} \phi\right\}+\partial_{33} \phi \\
& \quad+\varepsilon\left\{\beta_{3, \varepsilon} \partial_{3} \phi-i \sum_{j=1}^{2}\left[\frac{\alpha_{j, \varepsilon}}{\sqrt{G_{\varepsilon}}} \frac{\partial_{j} b_{j}}{\varepsilon^{2 / 3}}+\beta_{j, \varepsilon} b_{j}\right] \phi\right\} \\
& \quad+\varepsilon^{4 / 3} \sum_{j=1}^{2} \beta_{j, \varepsilon} \partial_{j} \phi+\varepsilon^{2} \mu \phi=0 .
\end{aligned}
$$

Note that

$$
\partial_{j} b_{j}(y, z)=O\left(\varepsilon^{2 / 3}\right), \quad j=1,2 .
$$

These computational results will be used in Section 4 . 


\section{$\S 4$. Upper bound of $\mu\left(\varepsilon^{-2} \mathbf{F}_{\mathbf{h}}\right)$ AND LOWER BOUnd of $H_{C_{3}}(\mathbf{h}, \mathbf{k})$}

Let the vector field $\mathbf{F}_{\mathbf{h}}$ satisfy (1.3). In this section we give an upper bound estimate for the lowest eigenvalue $\mu\left(\varepsilon^{-2} \mathbf{F}_{\mathbf{h}}\right)$, which is optimal up to the second term.

Theorem 4.1. Assume that $\Omega$ is a bounded 3-dimensional domain of class $C^{4}$. We have

$$
\mu\left(\varepsilon^{-2} \mathbf{F}_{\mathbf{h}}\right) \leq \frac{1}{\varepsilon^{2}}\left\{\beta_{0}+\varepsilon^{2 / 3} C_{2} P_{\min }+O\left(\varepsilon^{5 / 6}\right)\right\},
$$

where $P_{\min }$ is given in (1.5) and $C_{2}$ is given in (1.6).

Proof. Let us recall the definition of $(\partial \Omega)_{\mathbf{h}}$, the function $P(x)$ and $\mathcal{P}$ given in Section 1 . In the proof we write

$$
\mu_{p}=C_{2} P_{\min }=\lambda_{0}\left(1-\alpha_{0}\right)^{1 / 3} P_{\min } .
$$

We fix a point $X_{0} \in \mathcal{P}$, and in a neighborhood of $X_{0}$ we use the coordinates $(y, z)$ introduced in Section 3. To save notation, we will not distinguish a function in the original coordinates or in new coordinates. As in Section 3, we choose $\mathbf{F}_{\mathbf{h}}=\sum_{j=1}^{3} G^{j j} \hat{a}_{j} \partial_{j} \mathcal{F}$; here $\hat{a}_{j}$ 's are given in (3.9). For a function $\psi(x)$ with compact support, we have

$$
\begin{aligned}
& \int_{\Omega}\left|\nabla_{\varepsilon^{-2} \mathbf{F}_{\mathbf{h}}} \psi\right|^{2} d x=\int_{\mathbb{R}_{+}^{3}} \sum_{j=1}^{3} \sqrt{G(y, z)} G^{j j}(y, z)\left|\partial_{y_{j}} \psi-i \varepsilon^{-2} \hat{a}_{j} \psi\right|^{2} d y d z, \\
& \int_{\Omega}|\psi|^{2} d x=\int_{\mathbb{R}_{+}^{3}} \sqrt{G(y, z)}|\psi|^{2} d y d z .
\end{aligned}
$$

Here $d y=d y_{1} d y_{2}$. Let us define $G_{j j, \varepsilon}, G_{\varepsilon}$ and $b_{j}(y, z)$ as in Section 3 (see (3.12)), and choose a test function in the form

$$
\psi(x)=\phi\left(\frac{y}{\varepsilon^{2 / 3}}, \frac{z}{\varepsilon}\right) .
$$

We compute

$$
\mu\left(\varepsilon^{-2} \mathbf{F}_{\mathbf{h}}\right) \leq \frac{\int_{\Omega}\left|\nabla_{\varepsilon^{-2} \mathbf{F}_{\mathbf{h}}} \psi\right|^{2} d x}{\int_{\Omega}|\psi|^{2} d x}=\varepsilon^{-2} \frac{I[\phi]}{I I[\phi]},
$$

where

$$
\begin{aligned}
& I[\psi]=\int_{\mathbb{R}_{+}^{3}}\left\{\sqrt{\frac{G_{22, \varepsilon}}{G_{11, \varepsilon}}}\left|\varepsilon^{1 / 3} \partial_{y_{1}} \phi-i b_{1} \phi\right|^{2}+\sqrt{\frac{G_{11, \varepsilon}}{G_{22, \varepsilon}}}\left|\varepsilon^{1 / 3} \partial_{y_{2}} \phi-i b_{2} \phi\right|^{2}\right. \\
& \left.+\sqrt{G_{\varepsilon}}\left|\partial_{z} \phi\right|^{2}\right\} d y d z, \\
& I I[\psi]=\int_{\mathbb{R}_{+}^{3}} \sqrt{G_{\varepsilon}}|\phi|^{2} d y d z .
\end{aligned}
$$

Let us choose

$$
\begin{aligned}
& \phi(y, z)=\phi_{0}(y, z)+\varepsilon^{1 / 3} \phi_{1}(y, z), \\
& \text { where } \quad \phi_{0}(y, z)=w(y) u(z), \quad \phi_{1}(y, z)=w_{1}(y) u_{1}(z),
\end{aligned}
$$

where $u$ is a positive eigenfunction of (2.1) with $z=z_{0}$ and $\beta=\beta_{0}$, and $u_{1}$ is a solution of (2.3) for this $u$ (see Section 2); $w$ is to be determined later and $w_{1}$ is 
determined by $w$ through the equality

$$
w_{1}(y)=\frac{2}{\sqrt{g}}\left[i h_{2} \partial_{1} w-i h_{1} \partial_{2} w-\left(a_{1}^{(2)} h_{1} y_{1}^{2}+a_{2}^{(1)} h_{2} y_{2}^{2}\right) w\right]
$$

where $a_{2}^{(1)}$ and $a_{1}^{(2)}$ are given in (3.10). We also assume that $u, u_{1}, w$ and $w_{1}$ satisfy

$$
\begin{array}{ll}
\|u\|_{L^{2}\left(\mathbb{R}_{+}\right)}=1, & \left\|u_{1}\right\|_{L^{2}\left(\mathbb{R}_{+}\right)}=1, \\
\|w\|_{L^{2}\left(\mathbb{R}^{2}\right)}=1, & \left\|w_{1}\right\|_{L^{2}\left(\mathbb{R}^{2}\right)}=1, \\
\int_{0}^{+\infty} u(z) u_{1}(z) d z=0, & \int_{\mathbb{R}^{2}} y_{j}|w(y)|^{2} d y=0, \quad j=1,2 .
\end{array}
$$

Recall that $u$ and $u_{1}$ exponentially decay. So, in the following computations we can drop various small terms. In the following, $g_{j j}$, etc. denote the value of the involved functions at $X_{0}$. Using (3.5) and equation (3.13), we find

$$
\begin{aligned}
& \sqrt{\frac{G_{22, \varepsilon}}{G_{11, \varepsilon}}}\left|\varepsilon^{1 / 3} \partial_{y_{1}} \phi-i b_{1} \phi\right|^{2}=h_{2}^{2} \sqrt{\frac{g_{11}}{g_{22}}}\left(z+z_{0}\right)^{2}\left|\phi_{0}\right|^{2}+2 \varepsilon^{1 / 3} h_{2}\left(z+z_{0}\right) \Re\left(i \bar{\phi}_{0} R_{1}\right) \\
& +\varepsilon^{2 / 3}\left\{\sqrt{\frac{g_{22}}{g_{11}}}\left|R_{1}\right|^{2}+c_{j} h_{2}^{2} \sqrt{\frac{g_{11}}{g_{22}}}\left(z+z_{0}\right)^{2} y_{j}\left|\phi_{0}\right|^{2}+2 h_{2}\left(z+z_{0}\right) \Re\left(i \bar{\phi}_{0} R_{2}\right)\right\}+O(\varepsilon), \\
& \sqrt{\frac{G_{11, \varepsilon}}{G_{22, \varepsilon}}}\left|\varepsilon^{1 / 3} \partial_{y_{2}} \phi-i b_{2} \phi\right|^{2}=h_{1}^{2} \sqrt{\frac{g_{22}}{g_{11}}}\left(z+z_{0}\right)^{2}\left|\phi_{0}\right|^{2}-2 \varepsilon^{1 / 3} h_{1}\left(z+z_{0}\right) \Re\left(i \bar{\phi}_{0} Z_{1}\right) \\
& +\varepsilon^{2 / 3}\left\{\sqrt{\frac{g_{11}}{g_{22}}}\left|Z_{1}\right|^{2}-c_{j} h_{1}^{2} \sqrt{\frac{g_{22}}{g_{11}}}\left(z+z_{0}\right)^{2} y_{j}\left|\phi_{0}\right|^{2}-2 h_{1}\left(z+z_{0}\right) \Re\left(i \bar{\phi}_{0} Z_{2}\right)\right\}+O(\varepsilon), \\
& \sqrt{G_{\varepsilon}}\left|\partial_{z} \phi\right|^{2}=\sqrt{g}\left|\partial_{3} \phi_{0}\right|^{2}+2 \varepsilon^{1 / 3} \sqrt{g} \Re\left(\partial_{z} \phi_{0} \partial_{z} \bar{\phi}_{1}\right)+\varepsilon^{2 / 3} \sqrt{g}\left(d_{j} y_{j}\left|\partial_{z} \phi_{0}\right|^{2}+\left|\partial_{z} \phi_{1}\right|^{2}\right) \\
& +O(\varepsilon),
\end{aligned}
$$

where

$$
\begin{aligned}
& c_{j}=\frac{1}{2}\left(\frac{g_{22, j}}{g_{22}}-\frac{g_{11, j}}{g_{11}}\right), \\
& d_{j}=\frac{1}{2}\left(\frac{g_{11, j}}{g_{11}}+\frac{g_{22, j}}{g_{22}}\right), \quad j=1,2, \\
& R_{1}=\partial_{1} \phi_{0}+i a_{2}^{(1)} y_{2}^{2} \phi_{0}-i h_{2} \sqrt{\frac{g_{11}}{g_{22}}}\left(z+z_{0}\right) \phi_{1}, \\
& R_{2}=\partial_{1} \phi_{1}+i a_{2}^{(1)} y_{2}^{2} \phi_{1}-i\left(a_{13}^{(1)} y_{1}+a_{23}^{(1)} y_{2}\right)\left(z+z_{0}\right) \phi_{0}, \\
& Z_{1}=\partial_{2} \phi_{0}-i a_{1}^{(2)} y_{1}^{2} \phi_{0}+i h_{1} \sqrt{\frac{g_{22}}{g_{11}}}\left(z+z_{0}\right) \phi_{1}, \\
& Z_{2}=\partial_{2} \phi_{1}-i a_{1}^{(2)} y_{1}^{2} \phi_{1}-i\left(a_{13}^{(2)} y_{1}+a_{23}^{(2)} y_{2}\right)\left(z+z_{0}\right) \phi_{0} .
\end{aligned}
$$

We compute

$$
I[\phi]=\sqrt{g}\left\{I_{0}+2 \varepsilon^{1 / 3} I_{1}+\varepsilon^{2 / 3} I_{2}\right\}+O(\varepsilon),
$$


where

$$
\begin{aligned}
I_{0}= & \int_{\mathbb{R}_{+}^{3}}\left\{\left|\partial_{z} \phi_{0}\right|^{2}+\left(\frac{h_{1}^{2}}{g_{11}}+\frac{h_{2}^{2}}{g_{22}}\right)\left(z+z_{0}\right)^{2}\left|\phi_{0}\right|^{2}\right\} d y d z, \\
I_{1}= & \int_{\mathbb{R}_{+}^{3}}\left\{\Re\left(\partial_{z} \bar{\phi}_{0} \partial_{z} \phi_{1}\right)+\frac{z+z_{0}}{\sqrt{g}} \Re\left[i \bar{\phi}_{0}\left(h_{2} R_{1}-h_{1} Z_{1}\right)\right]\right\} d y d z, \\
I_{2}= & \int_{\mathbb{R}_{+}^{3}}\left\{\left|\partial_{z} \phi_{1}\right|^{2}+d_{j} y_{j}\left|\partial_{z} \phi_{0}\right|^{2}+\frac{1}{g_{11}}\left|R_{1}\right|^{2}+\frac{1}{g_{22}}\left|Z_{1}\right|^{2}\right. \\
& \left.+c_{j}\left(\frac{h_{2}^{2}}{g_{22}}-\frac{h_{1}^{2}}{g_{11}}\right) y_{j}\left(z+z_{0}\right)^{2}\left|\phi_{0}\right|^{2}+\frac{2\left(z+z_{0}\right)}{\sqrt{g}} \Re\left[i \bar{\phi}_{0}\left(h_{2} R_{2}-h_{1} Z_{2}\right)\right]\right\} d y d z .
\end{aligned}
$$

Recall that $h_{1}^{2} / g_{11}+h_{2}^{2} / g_{22}=1$, and

$$
\begin{aligned}
& \int_{0}^{+\infty}\left\{u^{\prime} u_{1}^{\prime}+\left(z+z_{0}\right)^{2} u u_{1}\right\} d z=\beta_{0} \int_{0}^{+\infty} u u_{1} d z=0 \\
& \int_{0}^{+\infty}\left\{\left|u_{1}^{\prime}\right|^{2}+\left(z+z_{0}\right)^{2} u_{1}^{2}\right\} d z=\beta_{0}\left\|u_{1}\right\|_{L^{2}\left(\mathbb{R}_{+}\right)}^{2}+\frac{\alpha_{0}}{4}
\end{aligned}
$$

see Section 2. Using (4.4) and (4.5) we find $I_{0}=\beta_{0}, I_{1}=0$, and

$$
\begin{aligned}
I_{2} & =\int_{0}^{+\infty}\left\{\left|u_{1}^{\prime}\right|^{2}+\left(z+z_{0}\right)^{2} u_{1}^{2}\right\} d z \int_{\mathbb{R}^{2}}\left|w_{1}\right|^{2} d y \\
& +\|u\|_{L^{2}\left(\mathbb{R}_{+}\right)}^{2} \int_{\mathbb{R}^{2}}\left\{\frac{1}{g_{11}}\left|\partial_{y_{1}} w+i a_{2}^{(1)} y_{2}^{2} w\right|^{2}+\frac{1}{g_{22}}\left|\partial_{y_{2}} w-i a_{1}^{(2)} y_{1}^{2} w\right|^{2}\right\} d y \\
& +\left\{d_{j}\left\|u^{\prime}\right\|_{L^{2}\left(\mathbb{R}_{+}\right)}^{2}+c_{j}\left(\frac{h_{2}^{2}}{g_{22}}-\frac{h_{1}^{2}}{g_{11}}\right) \int_{0}^{+\infty}\left(z+z_{0}\right)^{2} u^{2} d z\right\} \int_{\mathbb{R}^{2}} y_{j}|w|^{2} d y \\
& +\frac{2}{\sqrt{g}} \int_{0}^{+\infty}\left(z+z_{0}\right)^{2} u^{2} d z \int_{\mathbb{R}^{2}}\left[h_{2}\left(a_{13}^{(1)} y_{1}+a_{23}^{(1)} y_{2}\right)-h_{1}\left(a_{13}^{(2)} y_{1}+a_{23}^{(2)} y_{2}\right)\right]|w|^{2} d y \\
& +\frac{4}{\sqrt{g}} \int_{0}^{+\infty}\left(z+z_{0}\right) u u_{1} d z \int_{\mathbb{R}^{2}} \Re\left\{i \overline { w } _ { 1 } \left[h_{2}\left(\partial_{y_{1}} w+i a_{2}^{(1)} y_{2}^{2} w\right)\right.\right. \\
& =\beta_{0}\left\|u_{1}\right\|_{L^{2}\left(\mathbb{R}_{+}\right)}^{2}\left\|w_{1}\right\|_{L^{2}\left(\mathbb{R}^{2}\right)}^{2}+\frac{\alpha_{0}}{4}\left\|w_{1}\right\|_{L^{2}\left(\mathbb{R}^{2}\right)}^{2} \\
& +\int_{\mathbb{R}^{2}}\left\{\frac{1}{g_{11}}\left|\partial_{y_{1}} w+i a_{2}^{(1)} y_{2}^{2} w\right|^{2}+\frac{1}{g_{22}}\left|\partial_{y_{2}} w-i a_{1}^{(2)} y_{1}^{2} w\right|^{2}\right\} d y \\
& +\frac{4}{\sqrt{g}} \int_{0}^{+\infty}\left(z+z_{0}\right) u u_{1} d z \int_{\mathbb{R}^{2}} \Re\left\{i \bar{w}_{1}\left[h_{2}\left(\partial_{y_{1}} w+i a_{2}^{(1)} y_{2}^{2} w\right)\right]\right\} d y \\
& =\beta_{0}\left\|u_{1}\right\|_{L^{2}\left(\mathbb{R}_{+}\right)}^{2}\left\|w_{1}\right\|_{L^{2}\left(\mathbb{R}^{2}\right)}^{2}+\int_{\mathbb{R}^{2}}\left\{c_{11}\left|\partial_{y_{1}} w+i a_{2}^{(1)} y_{2}^{2} w\right|^{2}+c_{22}\left|\partial_{y_{2}} w-i a_{1}^{(2)} y_{1}^{2} w\right|^{2}\right. \\
& \left.+2 c_{12} \Re\left[\left(\partial_{y_{1}} w+i a_{2}^{(1)} y_{2}^{2} w\right)\left(\partial_{y_{2}} \bar{w}+i a_{1}^{(2)} y_{1}^{2} \bar{w}\right)\right]\right\} d y \\
& =\beta_{0}\left\|u_{1}\right\|_{L^{2}\left(\mathbb{R}_{+}\right)}^{2}\left\|w_{1}\right\|_{L^{2}\left(\mathbb{R}^{2}\right)}^{2}+p[w],
\end{aligned}
$$


where $p[w]$ is the functional defined in Section 2, and

$$
\begin{aligned}
c_{11}= & \frac{1}{g_{11}}\left(1-\alpha_{0} \frac{h_{2}^{2}}{g_{22}}\right), \\
c_{12}= & \frac{\alpha_{0} h_{1} h_{2}}{g_{11} g_{22}}, \\
c_{22}= & \frac{1}{g_{22}}\left(1-\alpha_{0} \frac{h_{1}^{2}}{g_{11}}\right), \\
f_{0}= & c_{11}\left(a_{2}^{(1)}\right)^{2} y_{2}^{4}-2 c_{12} a_{2}^{(1)} a_{1}^{(2)} y_{1}^{2} y_{2}^{2}+c_{22}\left(a_{1}^{(2)}\right)^{2} y_{1}^{4} \\
& \quad+2 i c_{12}\left(a_{1}^{(2)} y_{1}-a_{2}^{(1)} y_{2}\right), \\
f_{1}= & c_{12} a_{1}^{(2)} y_{1}^{2}-c_{11} a_{2}^{(1)} y_{2}^{2}, \\
f_{2}= & c_{22} a_{1}^{(2)} y_{1}^{2}-c_{12} a_{2}^{(1)} y_{2}^{2} .
\end{aligned}
$$

On the other hand, we have

$$
I I[\phi]=\sqrt{g}\left\{1+\varepsilon^{2 / 3}\left\|u_{1}\right\|_{L^{2}\left(\mathbb{R}_{+}\right)}^{2}\left\|w_{1}\right\|_{L^{2}\left(\mathbb{R}^{2}\right)}^{2}\right\}+O(\varepsilon) .
$$

Hence

$$
\frac{I[\phi]}{I I[\phi]}=I_{0}+\varepsilon^{2 / 3}\left\{I_{2}-I_{0}\left\|u_{1}\right\|_{L^{2}\left(\mathbb{R}_{+}\right)}^{2}\left\|w_{1}\right\|_{L^{2}\left(\mathbb{R}^{2}\right)}^{2}\right\}+O(\varepsilon)=I_{0}+\varepsilon^{2 / 3} p[w]+O(\varepsilon) .
$$

Now we look for a proper function $w$ which satisfies all the requirements needed in the above computations, and such that all the error terms of order $O(\varepsilon)$ in the estimates can be controlled. By careful analysis on each term of order $O(\varepsilon)$, we see that they can be controlled by the integral of the function $\varepsilon\left(|y|^{2}+|z|^{2}\right)|\phi|^{2}$. From Proposition 2.5 we know that $(2.8)$ has a bounded solution $\psi_{p}(y)$ given in (2.18). In the new coordinates $x^{\prime}=\left(x_{1}^{\prime}, x_{2}^{\prime}\right)$ determined by the transformations $(2.10)$ and (2.13), we have

$$
\left|\psi_{p}(y)\right|=c v\left(x_{1}^{\prime}\right)
$$

which depends only on $x_{1}^{\prime}$ (here, in order to avoid confusion, we write the new coordinates given by $(2.13)$ by $x^{\prime}=\left(x_{1}^{\prime}, x_{2}^{\prime}\right)$, instead of $\left.x\right)$. Moreover, $\psi_{p}(y)$ and its partial derivatives exponentially decay as $x_{1}^{\prime}$ goes to infinity. However, $\psi_{p}(y)$ does not decay in $x_{2}^{\prime}$ direction. Hence we choose $w$ in the following form:

$$
w(y)=c \psi_{p}(y) \eta\left(x_{2}^{\prime}\right),
$$

where $\eta\left(x_{2}^{\prime}\right) \in L^{2}(\mathbb{R})$ and is an even function. Then, using Remark 2.6 we have

$$
\int_{\mathbb{R}^{2}} y_{j}|w(y)|^{2} d y=0, \quad j=1,2 .
$$

Since $u(z)$ and $u_{1}(z)$ exponentially decay in $z$, and $\left|\psi_{p}(y)\right|=c v\left(x_{1}^{\prime}\right)$ exponentially decays in $x_{1}^{\prime}$, we find that

$$
\frac{I[\phi]}{I I[\phi]} \leq \beta_{0}+\varepsilon^{2 / 3} p[w]+M_{1} \varepsilon \int_{\mathbb{R}^{2}}|y|^{2}|w|^{2} d y+M_{2} \varepsilon .
$$

To determine the function $\eta$, we consider the following variational problem:

$$
\delta(\varepsilon)=\inf _{\phi \in W^{1,2}(\mathbb{R})} \frac{\int_{-\infty}^{+\infty}\left\{\left|\phi^{\prime}(t)\right|^{2}+M_{1} \varepsilon^{1 / 3} t^{2}|\phi(t)|^{2}\right\} d t}{\|\phi\|_{L^{2}(\mathbb{R})}^{2}} .
$$


By rescaling $s=\varepsilon^{1 / 12} t$, we see that $\delta(\varepsilon)=\varepsilon^{1 / 6} \delta_{0}$, where

$$
\delta_{0}=\inf _{\varphi \in W^{1,2}(\mathbb{R})} \frac{\int_{-\infty}^{+\infty}\left\{\left|\varphi^{\prime}(s)\right|^{2}+M_{1} s^{2}|\varphi(s)|^{2}\right\} d s}{\|\varphi\|_{L^{2}(\mathbb{R})}^{2}} .
$$

The minimizers $\varphi_{0}(s)$ of the latter problem (4.8) exist. They are even functions and exponentially decay in $s$. So the minimizers $\phi_{0}(t)$ of the former problem (4.7) exist, they are even functions and exponentially decay in $t$, and

$$
\phi_{0}(t)=\varphi_{0}(s)=\varphi_{0}\left(\varepsilon^{1 / 12} t\right) .
$$

Now we choose a cut-off function $\eta_{n}(t)$ such that, $\eta_{n}(t)$ is an even function, $\eta_{n}(t)=1$ if $|t|<n, \eta_{n}(t)=0$ if $|t|>2 n$, and $\left|\eta_{n}^{\prime}(t)\right| \leq 2 / n$. Then we choose a suitable integer $n_{\varepsilon}$, and let

$$
\begin{aligned}
& \eta\left(x_{2}^{\prime}\right)=c(\varepsilon) \eta_{n_{\varepsilon}}\left(x_{2}^{\prime}\right) \varphi_{0}\left(\varepsilon^{1 / 12} x_{2}^{\prime}\right), \\
& w(y)=c(\varepsilon) \psi_{p}(y) \eta\left(x_{2}^{\prime}\right),
\end{aligned}
$$

where $c(\varepsilon)$ is chosen so that the requirement $\|w\|_{L^{2}\left(\mathbb{R}^{2}\right)}=1$ is satisfied. Using this $w$, we defined the function $w_{1}$ by (4.3). Then we let

$$
\begin{aligned}
& \phi(y, z)=c(\varepsilon) \psi_{p}(y) \eta\left(x_{2}^{\prime}\right) u(z)+\varepsilon^{1 / 3} w_{1}(y) u_{1}(z), \\
& \psi(x)=\phi\left(\frac{y}{\varepsilon^{2 / 3}}, \frac{z}{\varepsilon}\right) .
\end{aligned}
$$

We have

$$
\begin{aligned}
& p[w]+M_{1} \varepsilon^{1 / 3} \int_{\mathbb{R}^{2}}|y|^{2}|w|^{2} d y \\
= & {\left[\mu_{p}+\delta(\varepsilon)+O(\varepsilon)\right]\|w\|_{L^{2}\left(\mathbb{R}^{2}\right)}^{2}=\left[\mu_{p}+O\left(\varepsilon^{1 / 6}\right)\right]\|w\|_{L^{2}\left(\mathbb{R}^{2}\right)}^{2} . }
\end{aligned}
$$

Hence

$$
\varepsilon^{2} \mu\left(\varepsilon^{-2} \mathbf{F}_{\mathbf{h}}\right) \leq \frac{I[\phi]}{I I[\phi]} \leq \beta_{0}+\varepsilon^{2 / 3}\left[\mu_{p}+O\left(\varepsilon^{1 / 6}\right)\right] \leq \beta_{0}+\varepsilon^{2 / 3} \mu_{p}+O\left(\varepsilon^{5 / 6}\right) .
$$

Theorem 4.2 (Lower bound estimate for $H_{C_{3}}$ ). Assume that $\Omega$ is a bounded 3dimensional domain of class $C^{4}$. Then for any unit vector $\mathbf{h}$ and large $\kappa$, we have

$$
H_{C_{3}}(\mathbf{h}, \kappa) \geq \frac{\kappa}{\beta_{0}}-\frac{C_{2}}{\beta_{0}^{5 / 3}} P_{\min } \kappa^{1 / 3}+O\left(\kappa^{2 / 9}\right) .
$$

Proof. Note that, if $\mu\left(\sigma \kappa \mathbf{F}_{\mathbf{h}}\right)<\kappa^{2}$, then the Ginzburg-Landau functional $\mathcal{E}$ has a nontrivial minimizer; see LP3] (Lemma 2.1). Thus, the lower bound of $H_{C_{3}}$ can be obtained by using the upper bound of the lowest eigenvalue $\mu\left(\sigma \kappa \mathbf{F}_{\mathbf{h}}\right)$ given in Theorem 4.1, and using the argument in [LP3].

\section{§5. Elliptic estimates}

In this section we establish some estimates for the minimizers of the GinzburgLandau functional $\mathcal{E}$. Throughout this section we assume that $\Omega$ is a bounded and simply-connected 3 -dimensional domain of class $C^{4}$. For convenience, we introduce

$$
\varepsilon=\frac{1}{\sqrt{\sigma \kappa}}, \quad \lambda_{\varepsilon}=\frac{\kappa}{\sigma} .
$$


Note that $\lambda_{\varepsilon}$ depends actually on both $\kappa$ and $\sigma$. From (1.4), $H_{C_{3}}(\mathbf{h}, \kappa)=\frac{\kappa}{\beta_{0}}+o(\kappa)$. We also believe that $H_{C_{2}}(\kappa, \mathbf{h}) \sim \kappa$ for large value of $\kappa$. Since we are considering applied fields in the range between $H_{C_{2}}$ and $H_{C_{3}}$, we assume in the following that

$$
\lim _{\varepsilon \rightarrow 0} \lambda_{\varepsilon}=\lambda, \quad \beta_{0} \leq \lambda \leq 1 .
$$

The functional $\mathcal{E}$ can be written as follows:

$$
\mathcal{E}[\psi, \mathbf{A}]=\int_{\Omega}\left\{\left|\nabla_{\varepsilon^{-2} \mathbf{A}} \psi\right|^{2}-\frac{\lambda_{\varepsilon}}{\varepsilon^{2}}|\psi|^{2}+\frac{\lambda_{\varepsilon}}{2 \varepsilon^{2}}|\psi|^{4}\right\} d x+\frac{1}{\varepsilon^{4}} \int_{\mathbb{R}^{3}}|\operatorname{curl} \mathbf{A}-\mathbf{h}|^{2} d x .
$$

Let $W^{1,2}(\Omega, \mathbb{C})$ be the Sobolev space of all complex-valued functions defined on $\bar{\Omega}$. Let

$$
\begin{aligned}
& \mathbf{D}^{1,2}\left(\mathbb{R}^{3}\right)=\left\{\mathbf{A} \in L_{\text {loc }}^{1}\left(\mathbb{R}^{3}\right):|\nabla \mathbf{A}| \in L^{2}\left(\mathbb{R}^{3}\right)\right\}, \\
& \mathbf{D}^{1,2}\left(\mathbb{R}^{3}, \operatorname{div}\right)=\left\{\mathbf{A} \in \mathbf{D}^{1,2}\left(\mathbb{R}^{3}\right): \operatorname{div} \mathbf{A}=0 \text { in } \mathbb{R}^{3}\right\} .
\end{aligned}
$$

Given a unit vector $\mathbf{h}$, let $\mathbf{F}_{\mathbf{h}}$ be the smooth vector field satisfying (1.3) and let

$$
\mathcal{W}(\Omega)=\left\{(\psi, \mathbf{A}): \phi \in W^{1,2}(\Omega, \mathbb{C}), \mathbf{A}-\mathbf{F}_{\mathbf{h}} \in \mathbf{D}^{1,2}\left(\mathbb{R}^{3}\right)\right\} .
$$

We consider the variational problem for $\mathcal{E}$ on $\mathcal{W}(\Omega)$, and define

$$
C(\varepsilon)=\inf _{(\psi, \mathbf{A}) \in \mathcal{W}(\Omega)} \mathcal{E}[\psi, \mathbf{A}] .
$$

It is easy to show that the minimizers exist. By the gauge invariance of $\mathcal{E}$, we can restrict the functional $\mathcal{E}$ on a subspace

$$
\mathcal{W}(\Omega, \operatorname{div})=\left\{(\psi, \mathbf{A}) \in \mathcal{W}(\Omega): \operatorname{div} \mathbf{A}=0 \text { in } \mathbb{R}^{3}\right\} .
$$

Then, the minimizers satisfy the Euler equation

$$
\begin{cases}-\nabla_{\varepsilon^{-2} \mathbf{A}}^{2} \psi=\frac{\lambda_{\varepsilon}}{\varepsilon^{2}}\left(1-|\psi|^{2}\right) \psi & \text { in } \Omega, \\ \operatorname{curl}^{2}\left(\mathbf{A}-\mathbf{F}_{\mathbf{h}}\right)=\varepsilon^{2} \Im\left(\bar{\psi} \nabla_{\varepsilon^{-2} \mathbf{A}} \psi\right) \chi_{\Omega} & \text { in } \mathbb{R}^{3}, \\ \left(\nabla_{\varepsilon^{-2} \mathbf{A}} \psi\right) \cdot \nu=0, & \text { on } \partial \Omega, \\ \mathbf{A}-\mathbf{F}_{\mathbf{h}} \in \mathbf{D}^{1,2}\left(\mathbb{R}^{3}, \operatorname{div}\right), & \end{cases}
$$

where $\chi_{\Omega}(x)=1$ for $x \in \Omega$ and $\chi_{\Omega}(x)=0$ for $x \notin \Omega$. (5.2) is a weak form of (1.2); see [P4], also see $\mathrm{L}$ (Chapter 5 , Section 4 ). It has been proved by many authors that the minimal solutions of (5.2) satisfy

$$
\|\psi\|_{L^{\infty}(\Omega)} \leq 1 .
$$

The regularity of the minimal solutions has been discussed in $\mathrm{P} 4$. In the following we shall always write a minimizer of $\mathcal{E}$ by $\left(\psi^{\varepsilon}, \mathbf{A}^{\varepsilon}\right)$ and assume $\operatorname{div} \mathbf{A}^{\varepsilon}=0$ in $\Omega$. We always let $C$ denote a generic positive constant that is independent of $\varepsilon$, but may vary from line to line. Let $B_{R}$ denote the open ball centered at the origin and with radius $R, B_{R}^{+}=\left\{y \in B_{R}: y_{3}>0\right\}$ and $\Gamma_{R}=\left\{\left(y_{1}, y_{2}, 0\right): y_{1}^{2}+y_{2}^{2}<R\right\}$. Let

$$
t(x)=\operatorname{dist}(x, \partial \Omega) .
$$

Lemma 5.1. Under the condition (5.1), $\psi^{\varepsilon} \in C^{2+\alpha}(\bar{\Omega})$ and $\mathbf{A}^{\varepsilon} \in C^{1+\alpha}(\bar{\Omega})$ for any $0<\alpha<1$. Moreover, we have the following estimates:

(1) There exists $C>0$ independent of $\varepsilon$ such that

$$
\begin{aligned}
& \left\|\mathbf{A}^{\varepsilon}-\mathbf{F}_{\mathbf{h}}\right\|_{L^{6}\left(\mathbb{R}^{3}\right)} \leq C \varepsilon\left\|\psi^{\varepsilon}\right\|_{L^{4}(\Omega)}^{2}, \\
& \left\|\operatorname{curl} \mathbf{A}^{\varepsilon}-\mathbf{h}\right\|_{L^{2}\left(\mathbb{R}^{3}\right)} \leq C \varepsilon\left\|\psi^{\varepsilon}\right\|_{L^{4}(\Omega)}^{2} .
\end{aligned}
$$


(2) For any $0<\alpha<1$, and for any $R>0$ such that $\bar{\Omega} \subset B_{R}$, there exist $C(\alpha, R)>0$ independent of $\varepsilon$ and $q=3 /(1-\alpha)$ such that, for all small $\varepsilon>0$,

$$
\left\|\nabla\left(\mathbf{A}^{\varepsilon}-\mathbf{F}_{\mathbf{h}}\right)\right\|_{C^{\alpha}\left(R_{R}\right)} \leq C(\alpha, R)\left\{\varepsilon\left\|\psi^{\varepsilon}\right\|_{L^{4}(\Omega)}^{2}+\varepsilon^{2}\left\|\bar{\psi}^{\varepsilon} \nabla_{\varepsilon^{-2} \mathbf{A}^{\varepsilon}} \psi^{\varepsilon}\right\|_{L^{q}(\Omega)}\right\} .
$$

The proof is similar to the proof of Lemma 3.3 in [P4 and hence is omitted here. In the next lemma we need the estimate of the solutions near boundary. In a neighborhood of a point $X_{0} \in \partial \Omega$ we introduce the local coordinates $(y, z)$ by the diffeomorphism $\mathcal{F}$ introduced in Section 3 such that $\mathcal{F}(0,0)=X_{0}$. We define $\tilde{\psi}^{\varepsilon}$ and $\tilde{\mathbf{A}}^{\varepsilon}$ as in (3.1):

$$
\begin{aligned}
& \tilde{\psi}^{\varepsilon}(y, z)=\psi^{\varepsilon}(\mathcal{F}(y, z)), \\
& \tilde{\mathbf{A}}^{\varepsilon}(y, z)=\mathbf{A}^{\varepsilon}(\mathcal{F}(y, z))=\sum_{j=1}^{3} G^{j j}(y, z) a_{j}^{\varepsilon}(y, z) \partial_{j} \mathcal{F}(y, z),
\end{aligned}
$$

where $y=\left(y_{1}, y_{2}\right), \partial_{1}=\partial_{y_{1}}, \partial_{2}=\partial_{y_{2}}, \partial_{3}=\partial_{z}, a_{j}^{\varepsilon}(y, z)=\mathbf{A}^{\varepsilon}(\mathcal{F}(y, z)) \cdot \partial_{j} \mathcal{F}(y, z)$. Then, since $\nu=-\partial_{3} \mathcal{F}$ on $\partial \Omega$ and $G_{12}=G_{13}=G_{23}=0, G_{33}=1$, we have

$$
\left\{\begin{aligned}
-\frac{1}{\sqrt{G}} \sum_{j=1}^{3}\left\{\partial_{j}\left(\sqrt{G} G^{j j} \partial_{j} \tilde{\psi}^{\varepsilon}\right)-2 \varepsilon^{-2} i \sqrt{G} G^{j j} a_{j}^{\varepsilon} \partial_{j} \tilde{\psi}^{\varepsilon}\right. & \\
\left.-\varepsilon^{-4} \sqrt{G} G^{j j}\left|a_{j}^{\varepsilon}\right|^{2} \tilde{\psi}^{\varepsilon}\right\}=\frac{\lambda_{\varepsilon}}{\varepsilon^{2}}\left(1-\left|\tilde{\psi}^{\varepsilon}\right|^{2}\right) \tilde{\psi}^{\varepsilon} & \text { in } B_{R_{0}}^{+} \\
\partial_{3} \tilde{\psi}^{\varepsilon}-i a_{3}^{\varepsilon} \tilde{\psi}^{\varepsilon}=0 & \text { on } \Gamma_{R_{0}} .
\end{aligned}\right.
$$

Lemma 5.2. Assume that (5.1) holds.

(1) There exist positive constants $C$ and $\varepsilon_{0}$, such that for any $0<\varepsilon<\varepsilon_{0}$ we have

$$
\left\|\nabla_{\varepsilon^{-2} \mathbf{A}^{\varepsilon}} \psi^{\varepsilon}\right\|_{C(\bar{\Omega})} \leq \frac{C}{\varepsilon} .
$$

(2) Given $0<\alpha<1$, there exist positive constants $C_{1}(\alpha)$ and $\varepsilon_{1}(\alpha)$, and $q=$ $3 /(1-\alpha)$, such that for any $0<\varepsilon<\varepsilon_{1}(\alpha)$,

$$
\left\|\nabla\left(\mathbf{A}^{\varepsilon}-\mathbf{F}_{\mathbf{h}}\right)\right\|_{C^{\alpha}(\bar{\Omega})} \leq \varepsilon C_{1}(\alpha)\left\{\left\|\psi^{\varepsilon}\right\|_{L^{4}(\Omega)}^{2}+\left\|\psi^{\varepsilon}\right\|_{L^{q}(\Omega)}\left\|\psi^{\varepsilon}\right\|_{L^{\infty}(\Omega)}\right\} .
$$

(3) Given $0<\alpha<2 \sqrt{1-\beta_{0}}$, there exist positive constants $C_{2}(\alpha)$ and $\varepsilon_{2}(\alpha)$, such that for all $0<\varepsilon<\varepsilon_{2}(\alpha)$,

$$
\int_{\Omega} \exp \left(\frac{\alpha}{\varepsilon} t(x)\right)\left\{\left|\psi^{\varepsilon}\right|^{2}+\varepsilon^{2}\left|\nabla_{\varepsilon^{-2} \mathbf{A}^{\varepsilon}} \psi^{\varepsilon}\right|^{2}\right\} d x \leq C_{2}(\alpha) \varepsilon .
$$

Proof. Note that, in the 2-dimensional case we have $C^{2+\alpha}$ estimates on the closed domain for the magnetic potential, while in the 3-dimensional case, we only have $\mathbf{A}^{\varepsilon} \in C_{\mathrm{loc}}^{3}(\Omega) \cap C^{1+\alpha}(\bar{\Omega})$ for any $0<\alpha<1$.

To prove conclusion (1), we modify the blow-up arguments that were used to study the 2-dimensional case in [LP3] (Proposition 3.1) and [HP] (Proposition 4.2). Let $X^{\varepsilon}$ be the maximum point of the function $\left|\nabla_{\varepsilon^{-2} \mathbf{A}^{\varepsilon}} \psi^{\varepsilon}\right|$. In the interior blow-up case, namely, if $\operatorname{dist}\left(X^{\varepsilon}, \partial \Omega\right) \gg \varepsilon$, then the argument of [HP] remains valid in the 3 -dimensional case and we find

$$
\left|\nabla_{\varepsilon^{-2} \mathbf{A}^{\varepsilon}} \psi^{\varepsilon}\left(X^{\varepsilon}\right)\right| \leq C \varepsilon^{-1} .
$$

Now let us consider the boundary blow-up case, namely, $\operatorname{dist}\left(X^{\varepsilon}, \partial \Omega\right) \leq C \varepsilon$ for all small $\varepsilon$. Choose $Q^{\varepsilon} \in \partial \Omega$ such that $\left|X^{\varepsilon}-Q^{\varepsilon}\right|=\operatorname{dist}\left(X^{\varepsilon}, \partial \Omega\right)$. Let $\mathcal{F}_{\varepsilon}(y, z)$ be a diffeomorphism which straightens a portion of $\partial \Omega$ surrounding $Q^{\varepsilon}$ as described 
in Section 3 such that $\mathcal{F}_{\varepsilon}(0,0)=Q^{\varepsilon}$. Similar to (5.3) we define $\tilde{\psi}^{\varepsilon}(y, z)$ and $\tilde{\mathbf{A}}^{\varepsilon}(y, z)$, with $\mathcal{F}$ replaced by $\mathcal{F}_{\varepsilon}$. Then $\tilde{\psi}^{\varepsilon}$ satisfies an equation in the form of (5.4), with coefficients depending on $\mathcal{F}_{\varepsilon}$ instead of depending on $\mathcal{F}$. We make gauge transformation and rescaling

$$
\begin{aligned}
& \tilde{\phi}_{\varepsilon}(y, z)=e^{-i \chi_{\varepsilon}} \tilde{\psi}^{\varepsilon}(\varepsilon y, \varepsilon z), \\
& \tilde{\mathbf{A}}_{\varepsilon}(y, z)=\frac{1}{\varepsilon}\left[\tilde{\mathbf{A}}^{\varepsilon}(\varepsilon y, \varepsilon z)-\tilde{\mathbf{A}}^{\varepsilon}(0,0)\right],
\end{aligned}
$$

where $\chi_{\varepsilon}$ is chosen properly; see [HP] (Proposition 4.2). Then $\tilde{\phi}_{\varepsilon}$ satisfies an elliptic equation in the form

$$
\begin{cases}-\Delta_{\varepsilon} \tilde{\phi}_{\varepsilon}=\lambda_{\varepsilon}\left(1-\left|\tilde{\phi}_{\varepsilon}\right|^{2}\right) \tilde{\phi}_{\varepsilon} & \text { in } B_{R_{0} / \varepsilon}^{+}, \\ \partial_{y_{3}} \tilde{\phi}_{\varepsilon}=i \varepsilon a_{\varepsilon, 3} \tilde{\phi}_{\varepsilon} & \text { on } \Gamma_{R_{0} / \varepsilon}\end{cases}
$$

where $\Delta_{\varepsilon}$ is the representation of $\nabla_{\mathbf{A}_{\varepsilon}}^{2}$ in the coordinates $(y, z)$, and $\mathbf{A}_{\varepsilon}(x)$ is a vector field that corresponds in the coordinates $x$ to $\tilde{\mathbf{A}}_{\varepsilon}$. Repeating the proof of Lemma 5.1 (see [P4], Lemma 3.3) we find that $\left\{\tilde{\mathbf{A}}_{\varepsilon}\right\}$ is bounded in $C_{\text {loc }}^{1+\alpha}\left(\mathbb{R}_{+}^{3}\right)$. Applying the Hölder estimate to the above equation we find that $\left\{\tilde{\phi}_{\varepsilon}\right\}$ is bounded in $C_{\text {loc }}^{2+\alpha}\left(\mathbb{R}_{+}^{3}\right)$. So $\left|\nabla_{\tilde{\mathbf{A}}_{\varepsilon}} \tilde{\psi}^{\varepsilon}(0,0)\right| \leq C$, and hence $\left|\nabla_{\varepsilon^{-2} \mathbf{A}^{\varepsilon}} \psi^{\varepsilon}\left(X^{\varepsilon}\right)\right| \leq C \varepsilon^{-1}$.

Conclusion (2) follows from conclusion (1) and Lemma 5.1. Conclusion (3) can be proved by using (1) and (2). We omit the details but refer to [HP] (Lemma 3.2) and [P1] (Lemma 7.2) for similar discussions in the 2-dimensional case.

Conclusion (3) of Lemma 5.2 describes in the $L^{2}$ sense that, under condition (5.1), the order parameters concentrate in a thin layer at boundary, and decay exponentially in the normal direction away from the boundary. It means that, under the applied field above $H_{C_{2}}$, superconductivity persists within a thin sheath near surface with thickness $O\left(\kappa^{-1}\right)$. More precise information of the concentration behavior of the order parameters will be given in Section 6. From conclusion (3) we also find that, for any nonnegative integer $k$,

$$
\int_{\Omega} t(x)^{k}\left\{\left|\psi^{\varepsilon}\right|^{2}+\varepsilon^{2}\left|\nabla_{\varepsilon^{-2} \mathbf{A} \varepsilon} \psi^{\varepsilon}\right|^{2}\right\} d x \leq C_{k} \varepsilon^{k+1} .
$$

In particular, we have

$$
\int_{\Omega}\left|\psi^{\varepsilon}\right|^{2} d x \leq C \varepsilon
$$

Let, for $q>3$,

$$
d(\varepsilon)=\left\|\psi^{\varepsilon}\right\|_{L^{4}(\Omega)}^{2}+\left\|\psi^{\varepsilon}\right\|_{L^{q}(\Omega)}\left\|\psi^{\varepsilon}\right\|_{L^{\infty}(\Omega)} .
$$

From (5.7) we find

$$
d(\varepsilon) \leq C\left\{\varepsilon^{1 / 2}\left\|\psi^{\varepsilon}\right\|_{L^{\infty}(\Omega)}+\varepsilon^{1 / q}\left\|\psi^{\varepsilon}\right\|_{L^{\infty}(\Omega)}^{2(q-1) / q}\right\} .
$$

Now we recall the function $\lambda(\vartheta)$ given in Lemma 2.3 , and the function $\varphi_{\mathbf{h}}(x)$ given in Remark 2.4. 
Lemma 5.3. Assume condition (5.1). There exists a constant $C>0$ such that, for any smooth function $\chi$ and small $\varepsilon>0$, we have

$$
\begin{aligned}
\int_{\Omega}\left|\nabla_{\varepsilon^{-2} \mathbf{A}^{\varepsilon}}\left(\chi \psi^{\varepsilon}\right)\right|^{2} d x \geq \varepsilon^{-2} & \int_{\Omega} W_{\mathbf{h}}(x)\left|\chi \psi^{\varepsilon}\right|^{2} d x \\
& -C \varepsilon^{-4 / 3} d(\varepsilon)^{2 / 3} \int_{\Omega}\left|\chi \psi^{\varepsilon}\right|^{2} d x
\end{aligned}
$$

where $d(\varepsilon)$ is given in (5.8) and

$$
W_{\mathbf{h}}(x)= \begin{cases}1-C \varepsilon^{1 / 2} & \text { if } \operatorname{dist}(x, \partial \Omega)>2 \varepsilon^{1 / 4} \\ \lambda\left(\varphi_{\mathbf{h}}(x)\right)-C \varepsilon^{1 / 2} & \text { if } \operatorname{dist}(x, \partial \Omega) \leq 2 \varepsilon^{1 / 4}\end{cases}
$$

Proof. The function $W_{\mathbf{h}}(x)$ was given by Helffer-Morame in HM2] 5 and it was given in [HM2] (Theorem 4.3) that, for any small $\varepsilon>0$ and for any $\phi \in W^{1,2}(\Omega)$, the following inequality holds:

$$
\int_{\Omega}\left|\nabla_{\varepsilon^{-2} \mathbf{F}_{\mathbf{h}}} \phi\right|^{2} d x \geq \frac{1}{\varepsilon^{2}} \int_{\Omega} W_{\mathbf{h}}(x)|\phi|^{2} d x .
$$

To prove (5.12) one applies the local analysis techniques developed in [HM1], and uses the upper bound of $\mu\left(\varepsilon^{-2} \mathbf{F}_{\mathbf{h}}\right)$ given in (1.13).

Lemma 5.3 can be proved exactly as in P3] (proof of Theorem 6.2, Step 2), and the details are omitted here. Note that, a result in the 2-dimensional case similar to $(5.10)$ has been proved in $\mathrm{HP}$, and in the proof the fact that we can choose a gauge such that the magnetic potential vanishes at the boundary makes it easy to control the error term; see (3.3) in $\mathrm{HP}$. In the 3-dimensional case, however, the magnetic potentials do not vanish at boundary, and harder estimates including the decomposition of vector fields near boundary are needed. Then we can apply (5.12) and the local analysis techniques in HM1 to prove (5.10). For more details see [P3].

Lemma 5.4. Let $\phi^{\varepsilon}$ be the eigenfunction of the operator $-\nabla_{\varepsilon^{-2} \mathbf{A}^{\varepsilon}}^{2}$ associated with the lowest eigenvalue $\mu\left(\varepsilon^{-2} \mathbf{A}^{\varepsilon}\right)$. Then (5.5) and (5.10) hold for $\phi^{\varepsilon}$.

Proof. The conclusion that the eigenfunctions $\phi^{\varepsilon}$ satisfy (5.5) was given in HM2], and it can be proved exactly as for $\psi^{\varepsilon}$. Note that (5.10) holds for any function satisfying (5.5), and hence is true for $\phi^{\varepsilon}$.

Theorem 5.5. Assume that $\Omega$ is a bounded and simply-connected 3-dimensional domain of class $C^{4}$. Given a unit vector $\mathbf{h}$ we have, for large $\kappa$,

$$
H_{C_{3}}(\mathbf{h}, \kappa) \leq \frac{\kappa}{\beta_{0}}+M \kappa^{1 / 2},
$$

where $M$ is a constant independent of $\mathbf{h}$ and $\kappa$.

Proof. We consider an applied field $\mathcal{H}=\sigma \mathbf{h}$ that is close to $H_{C_{3}}(\mathbf{h}, \kappa)$. From (1.4), we can choose $\sigma \sim \kappa / \beta_{0}$ as $\kappa \rightarrow \infty$. Hence $\varepsilon=(\sigma \kappa)^{-1 / 2} \sim \sqrt{\beta_{0}} \kappa^{-1}$. Let $\left(\psi^{\varepsilon}, \mathbf{A}^{\varepsilon}\right)$ be a nontrivial minimizer of the functional $\mathcal{E}$. We show that

$$
\mu\left(\varepsilon^{-2} \mathbf{A}^{\varepsilon}\right) \geq \frac{1}{\varepsilon^{2}}\left(\beta_{0}-M_{1} \varepsilon^{1 / 2}\right),
$$

\footnotetext{
${ }^{5}$ The notation $\vartheta$ and $\sigma(\vartheta)$ used in [HM2] are related to our notation $\varphi_{\mathbf{h}}$ and $\lambda\left(\varphi_{\mathbf{h}}\right)$ through $\vartheta=\frac{\pi}{2}-\varphi_{\mathbf{h}}, \sigma(\vartheta)=\lambda\left(\frac{\pi}{2}-\varphi_{\mathbf{h}}\right)$.
} 
where $M_{1}$ is a constant independent of $\varepsilon$ and $\mathbf{h}$. To prove (5.14), we let $\phi^{\varepsilon}$ be an eigenfunction of $-\nabla_{\varepsilon^{-2} \mathbf{A}^{\varepsilon}}^{2}$ associated with $\mu\left(\varepsilon^{-2} \mathbf{A}^{\varepsilon}\right)$. From Lemma 5.4 we have

$$
\int_{\Omega}\left|\nabla_{\varepsilon^{-2} \mathbf{A}^{\varepsilon}} \phi^{\varepsilon}\right|^{2} d x \geq \varepsilon^{-2} \int_{\Omega} W_{\mathbf{h}}(x)\left|\phi^{\varepsilon}\right|^{2} d x-C \varepsilon^{-4 / 3} d(\varepsilon)^{2 / 3} \int_{\Omega}\left|\phi^{\varepsilon}\right|^{2} d x .
$$

For the applied field close to $H_{C_{3}}$ we have $\left\|\psi^{\varepsilon}\right\|_{L^{\infty}(\Omega)}=o(1)$. From (5.9) we find $d(\varepsilon)=o\left(\varepsilon^{1 / q}\right)$ for $q>3$. From (5.11), we have

$$
\mu\left(\varepsilon^{-2} \mathbf{A}^{\varepsilon}\right) \geq \frac{1}{\varepsilon^{2}}\left\{\beta_{0}-C \varepsilon^{1 / 2}-o(1) \varepsilon^{2(1+q) /(3 q)}\right\} .
$$

By choosing $q<6$ in the above inequality we get (5.14).

Since $\psi^{\varepsilon} \not \equiv 0$, from [LP3] (Lemma 2.1) we know that $\mu\left(\varepsilon^{-2} \mathbf{A}^{\varepsilon}\right)<\kappa^{2}$. Now we have $\varepsilon^{-2}\left(\beta_{0}-M_{1} \varepsilon^{1 / 2}\right)<\kappa^{2}$. So

$$
\sigma<\frac{\kappa}{\beta_{0}-M_{1} \varepsilon^{1 / 2}}=\frac{\kappa}{\beta_{0}}\left[1+\frac{M_{1}}{\beta_{0}} \varepsilon^{1 / 2}+O(\varepsilon)\right] \leq \frac{\kappa}{\beta_{0}}+M_{2} \sqrt{\kappa} .
$$

Thus (5.13) is true. To show that $M$ can be chosen independent of $\mathbf{h}$, we replace $\mathbf{h}$ by a unit vector $\mathbf{h}_{\varepsilon}$ which depends on $\varepsilon$, and repeat the above discussion.

Proof of Theorem 1. Theorem 4.2 + Theorem 5.5.

\section{$\S 6$. Surface NuCleation}

In this section we study the nucleation of superconductivity of a bounded 3dimensional superconductor with large $\kappa$ and subjected to an applied field lying inbetween $H_{C_{2}}$ and $H_{C_{3}}$. We shall use Agmon's idea $\underline{\mathrm{A}}$ and the techniques developed in $[\mathrm{H}]$, and adapt the arguments from $[\mathrm{HP}]$. For this purpose we shall modify $\lambda\left(\varphi_{\mathbf{h}}(x)\right)-\beta_{0}$ to get a smooth function which measures the tangential distance from $(\partial \Omega)_{\mathbf{h}}$, where $\lambda(\vartheta)$ and $\varphi_{\mathbf{h}}(x)$ are given in Lemma 2.3 and Remark 2.4 6

Lemma 6.1. Fix a small $\delta>0$. There exists a function $\zeta_{\mathbf{h}}$ with the following properties:

(1) $\zeta_{\mathbf{h}} \in C^{1}(\bar{\Omega}) ; \zeta_{\mathbf{h}}(x) \geq 0$ on $\bar{\Omega}, \zeta_{\mathbf{h}}(x)=0$ on $(\partial \Omega)_{\mathbf{h}}$, and $\zeta_{\mathbf{h}}(x)>0$ on $(\partial \Omega) \backslash(\partial \Omega)_{\mathbf{h}}$.

(2) If $x \in \Omega(\delta)$ and $\delta \leq \varphi_{\mathbf{h}}(x) \leq \pi-\delta$, then $\zeta_{\mathbf{h}}(x)=\zeta_{0}\left(\varphi_{\mathbf{h}}(x)\right)$, where

$$
\zeta_{0}(\varphi)=\left|\int_{\varphi}^{\pi / 2}\left[\lambda(\vartheta)-\beta_{0}\right]^{1 / 2} d \vartheta\right|
$$

(3) There exists a positive constant $C_{\delta}$ depending only on $\Omega, \mathbf{h}$ and $\delta$, such that

$$
\left|\nabla \zeta_{\mathbf{h}}(x)\right| \leq C_{\delta}\left[\lambda\left(\varphi_{\mathbf{h}}(x)\right)-\beta_{0}\right]^{1 / 2} \text { on } \bar{\Omega} .
$$

(4) For $\varphi$ close to $\pi / 2$ we have the following estimate for the function $\zeta_{0}(\varphi)$ :

$$
\frac{1}{2} \sqrt{1-\beta_{0}}\left(\varphi-\frac{\pi}{2}\right)^{2}+O\left(\left(\varphi-\frac{\pi}{2}\right)^{4}\right) \leq \zeta_{0}(\varphi) \leq \frac{2}{3}\left|\varphi-\frac{\pi}{2}\right|^{3 / 2}+O\left(\left|\varphi-\frac{\pi}{2}\right|^{5 / 2}\right) .
$$

\footnotetext{
${ }^{6}$ We cannot simply take $\lambda\left(\varphi_{\mathbf{h}}(x)\right)-\beta_{0}$ to measure the tangential distance from $(\partial \Omega)_{\mathbf{h}}$, because $\varphi_{\mathbf{h}}(x)$ is not differentiable on $(\partial \Omega)_{\mathbf{h}}$, and we only know that $\lambda(\vartheta)$ is differentiable almost everywhere but we do not know yet whether $\lambda^{\prime}(\vartheta) \in L^{\infty}[0, \pi]$.
} 
Proof. Recall that $\lambda(\vartheta)$ is continuous for $\vartheta \in[0, \pi]$ and $\beta_{0} \leq \lambda(\vartheta) \leq 1$. Thus $\zeta_{0}$ is a $C^{1}$ function on $[0, \pi]$. We first define $\zeta_{\mathbf{h}}(x)=\zeta_{0}\left(\varphi_{\mathbf{h}}(x)\right)$ on $\{x \in \Omega(\delta): \delta \leq$ $\varphi_{\mathbf{h}}(x) \leq \pi-\delta$, and then extend it over $\bar{\Omega}$ to get a $C^{1}$ function. It is easy to check that $\zeta_{\mathbf{h}}$ satisfies (1), (2), (3). To verify (4), we use (2.5). For $0<\varphi<\pi / 2$ we have

$$
\begin{aligned}
\zeta_{0}(\varphi) & \geq \int_{\varphi}^{\pi / 2} \sqrt{1-\beta_{0}} \cos \vartheta d \vartheta=\sqrt{1-\beta_{0}}\left[1-\cos \left(\frac{\pi}{2}-\varphi\right)\right] \\
& =\frac{1}{2} \sqrt{1-\beta_{0}}\left(\frac{\pi}{2}-\varphi\right)^{2}+O\left(\left(\frac{\pi}{2}-\varphi\right)^{4}\right) .
\end{aligned}
$$

Now if $\pi / 2-\varphi>0$ is small, we have

$$
\begin{aligned}
\zeta_{0}(\varphi) & \leq \int_{\varphi}^{\pi / 2}\left[\beta_{0}(\sin \vartheta-1)+\cos \vartheta\right]^{1 / 2} d \vartheta=\int_{0}^{\pi / 2-\varphi}\left[\beta_{0}(\cos t-1)+\sin t\right]^{1 / 2} d t \\
& =\int_{0}^{\pi / 2-\varphi}\left[t-\frac{\beta_{0}}{2} t^{2}+O\left(t^{3}\right)\right]^{1 / 2} d t=\frac{2}{3}\left(\frac{\pi}{2}-\varphi\right)^{3 / 2}+O\left(\left(\frac{\pi}{2}-\varphi\right)^{5 / 2}\right) .
\end{aligned}
$$

Similarly, given $\beta_{0}<b<1$, we introduce $\zeta_{\mathbf{h}, b}(x)$ to measure the tangential distance from the set $\left\{x \in \partial \Omega: \lambda\left(\varphi_{\mathbf{h}}(x)\right) \leq b\right\}$. Recall that $\lambda(\vartheta)$ is strictly decreasing on $[0, \pi / 2]$. So the inverse function $\lambda^{-1}(b)$ exists and is continuous for $b \in\left[\beta_{0}, 1\right]$, and $\lambda^{-1}\left(\beta_{0}\right)=\pi / 2$. In the following we use the notation $a_{+}=$ $\max \{a, 0\}$.

Lemma 6.2. Given a unit vector $\mathbf{h}$ and a number $b \in\left(\beta_{0}, 1\right)$, for small $\delta>0$, there exists a function $\zeta_{\mathbf{h}, b} \in C(\bar{\Omega})$ with the following properties:

(1) $\zeta_{\mathbf{h}, b}(x) \geq 0$ on $\bar{\Omega}, \quad \zeta_{\mathbf{h}, b}(x)=0$ on $\left\{x \in \partial \Omega: \lambda\left(\varphi_{\mathbf{h}}(x)\right) \leq b\right\}$, and $\zeta_{\mathbf{h}, b}(x)>$ 0 on $\partial \Omega \backslash\left\{x \in \partial \Omega: \lambda\left(\varphi_{\mathbf{h}}(x)\right) \leq b\right\} ; \nabla \zeta_{\mathbf{h}, b}(x)$ exists almost everywhere and $\left|\nabla \zeta_{\mathbf{h}, b}(x)\right| \in L^{\infty}(\Omega)$

(2) If $x \in \Omega(\delta)$ and $\delta \leq \varphi_{\mathbf{h}}(x) \leq \pi-\delta$, then $\zeta_{\mathbf{h}, b}(x)=\zeta_{b}\left(\varphi_{\mathbf{h}}(x)\right)$, where

$$
\zeta_{b}(\varphi)=\int_{\varphi}^{\lambda^{-1}(b)}[\lambda(\vartheta)-b]^{1 / 2} d \vartheta \quad \text { if } 0 \leq \varphi \leq \lambda^{-1}(b),
$$

$\zeta_{b}(\varphi)=0$ if $\lambda^{-1}(b)<\varphi \leq \pi / 2$, and $\zeta_{b}(\varphi)=\zeta_{b}(\pi-\varphi)$ if $\pi / 2<\varphi \leq \pi$.

(3) There exists $C_{\delta, b}>0$ depending only on $\Omega, \mathbf{h}, \delta$ and $b$, such that

$$
\left|\nabla \zeta_{\mathbf{h}, b}(x)\right| \leq C_{\delta, b}\left[\left(\lambda\left(\varphi_{\mathbf{h}}(x)\right)-b\right)_{+}\right]^{1 / 2} .
$$

(4) We have the following estimate for the function $\zeta_{b}(\varphi)$ : For any fixed $l>0$, there exists $c_{l}(\varepsilon)$ satisfying $c_{l}(\varepsilon) \rightarrow 0$ as $\varepsilon \rightarrow 0$, such that, if $\lambda(\varphi) \leq b+l \varepsilon^{1 / 2}$, then

$$
0 \leq \zeta_{b}(\varphi) \leq c_{l}(\varepsilon) \varepsilon^{1 / 4}
$$

Proof. We only need to prove (4), and we only consider the case where $0<\varphi \leq \pi / 2$. Assume $\lambda(\varphi) \leq b+l \varepsilon^{1 / 2}$. Then $\varphi \geq \lambda^{-1}\left(b+l \varepsilon^{-1 / 2}\right)$. Since $\lambda(\vartheta)$ is strictly decreasing in $[0, \pi / 2]$, we have

$$
\begin{aligned}
\zeta_{b}(\varphi) & =\int_{\varphi}^{\lambda^{-1}(b)}[\lambda(\vartheta)-b]^{1 / 2} d \vartheta \leq\left[\lambda^{-1}(b)-\varphi\right][\lambda(\varphi)-b]^{1 / 2} \\
& \leq\left[\lambda^{-1}(b)-\varphi\right] l^{1 / 2} \varepsilon^{1 / 4} \leq\left[\lambda^{-1}(b)-\lambda^{-1}\left(b+l \varepsilon^{1 / 2}\right)\right] l^{1 / 2} \varepsilon^{1 / 4}=c_{l}(\varepsilon) \varepsilon^{1 / 4}
\end{aligned}
$$


where

$$
c_{l}(\varepsilon)=l^{1 / 2}\left[\lambda^{-1}(b)-\lambda^{-1}\left(b+l \varepsilon^{1 / 2}\right)\right]
$$

and $c_{l}(\varepsilon) \rightarrow 0 \quad$ as $\varepsilon \rightarrow 0$.

Theorem 6.3. Let $\Omega$ be a bounded, smooth and simply-connected domain in $\mathbb{R}^{3}$ and $\mathbf{h}$ a unit vector. Under the applied field $\mathcal{H}=\sigma \mathbf{h}$, the order parameters $\psi$ have the following concentration behavior for large $\kappa$ :

(1) If $\sigma=H_{C_{3}}(\mathbf{h}, \kappa)-a_{1} \kappa^{1 / 2}$ where $a_{1}>0$, there exist positive constants $\alpha_{1}$, $c_{1}, M_{1}$ such that

$$
\int_{\Omega} \exp \left(\alpha_{1} \kappa\left[\zeta_{\mathbf{h}}(x)-c_{1} \kappa^{-3 / 8}\right]\right)|\psi|^{2} d x \leq M_{1} \kappa^{1 / 2} \int_{\Omega\left(\kappa^{-1}\right)}|\psi|^{2} d x .
$$

In particular, there exist positive constants $\eta$ and $\gamma_{1}$ such that

$$
\int_{\Omega \backslash \mathcal{S}_{\kappa}(\mathbf{h})}|\psi|^{2} d x \leq M_{1} \exp \left(-\gamma_{1} \kappa^{5 / 8}\right) \int_{\Omega\left(\kappa^{-1}\right)}|\psi|^{2} d x,
$$

where

$$
\mathcal{S}_{k}(\mathbf{h})=\Omega\left(\kappa^{-1}\right) \cap\left\{\left|\varphi_{\mathbf{h}}(x)-\pi / 2\right| \leq \eta \kappa^{-3 / 16}\right\} .
$$

(2) If $\sigma=\kappa / b$, where $\beta_{0}<b<1$, there exist positive constants $\alpha_{2}, c_{2}$ and $M_{2}$, all depending on $b$, such that

$$
\int_{\Omega} \exp \left(\alpha_{2} \kappa\left[\zeta_{\mathbf{h}, b}(x)-c_{2} \kappa^{-1 / 4}\right]\right)|\psi|^{2} d x \leq M_{2} \kappa^{1 / 2} \int_{\Omega\left(\kappa^{-1}\right)}|\psi|^{2} d x .
$$

In particular, there exist positive constants $\gamma_{2}$ and $\eta(\kappa)$ depending on $b$, and $\vartheta(b)=$ $\lambda^{-1}(b)$, where $\eta(\kappa) \rightarrow 0$ as $\kappa \rightarrow \infty$, such that

$$
\int_{\Omega \backslash \mathcal{S}_{b, \kappa}(\mathbf{h})}|\psi|^{2} d x \leq M_{2} \exp \left(-\gamma_{2} \kappa^{3 / 4}\right) \int_{\Omega\left(\kappa^{-1}\right)}|\psi|^{2} d x,
$$

where

$$
\mathcal{S}_{b, \kappa}(\mathbf{h})=\Omega\left(\kappa^{-1}\right) \cap\left\{\vartheta(b)-\eta(\kappa) \leq \varphi_{\mathbf{h}}(x) \leq \pi-\vartheta(b)+\eta(\kappa)\right\} .
$$

Proof. As in Section 5, we let $\varepsilon=1 / \sqrt{\sigma \kappa}$ and $\lambda_{\varepsilon}=\kappa / \sigma$, and let $\left(\psi^{\varepsilon}, \mathbf{A}^{\varepsilon}\right)$ denote the minimal solutions of (5.2). Let

$$
\eta=\exp \left(\alpha \varepsilon^{-1} \zeta\right)
$$

where $\alpha$ is a positive constant and $\zeta$ is a real-valued $C^{1}$ function, both to be determined later. We multiply the first equation in (5.2) by $\eta^{2} \bar{\psi}^{\varepsilon}$ and integrate, and use (5.10) to get

$$
\begin{aligned}
0 & =\int_{\Omega}\left\{\left|\nabla_{\varepsilon^{-2} \mathbf{A}^{\varepsilon}}\left(\eta \psi^{\varepsilon}\right)\right|^{2}-|\nabla \eta|^{2}\left|\psi^{\varepsilon}\right|^{2}-\frac{\lambda_{\varepsilon}}{\varepsilon^{2}}\left(1-\left|\psi^{\varepsilon}\right|^{2}\right)\left|\eta \psi^{\varepsilon}\right|^{2}\right\} d x \\
& \geq \varepsilon^{-2} \int_{\Omega}\left\{W_{\mathbf{h}}(x)-\alpha^{2}|\nabla \zeta|^{2}-\lambda_{\varepsilon}+o\left(\varepsilon^{2 / 3}\right)\right\}\left|\eta \psi^{\varepsilon}\right|^{2} d x
\end{aligned}
$$

Let

$$
\rho=H_{C_{3}}(\mathbf{h}, \kappa)-\sigma, \quad C_{3}=C_{2} \beta_{0}^{-5 / 3} P_{\min } .
$$

From Theorem 1 we have

$$
\sigma=H_{C_{3}}(\mathbf{h}, \kappa)-\rho \geq \frac{\kappa}{\beta_{0}}\left\{1-\beta_{0} \kappa^{-1} \rho-C_{3} \beta_{0} \kappa^{-2 / 3}+O\left(\kappa^{-7 / 9}\right)\right\} .
$$


If $\kappa \gg \rho \geq c_{0} \kappa^{1 / 3}$, we have

$$
\lambda_{\varepsilon}=\frac{\kappa}{\sigma} \leq \beta_{0}+\beta_{0}^{2} \kappa^{-1} \rho+C_{3} \beta_{0}^{2} \kappa^{-2 / 3}+O\left(\kappa^{-7 / 9}\right)+O\left(\rho^{2} \kappa^{-2}\right) .
$$

Step 1. In case (1) we have

$$
\varepsilon=\sqrt{\beta_{0}} \kappa^{-1}+O\left(\kappa^{-3 / 2}\right), \quad \beta_{0}^{2} \kappa^{-1} \rho=d \varepsilon^{1 / 2}
$$

for some $d>0$. Then from (6.7),

$$
\lambda_{\varepsilon} \leq \beta_{0}+d \varepsilon^{1 / 2}+O\left(\varepsilon^{2 / 3}\right) .
$$

Let $\zeta=\zeta_{\mathbf{h}}$ and choose $\alpha>0$ such that $\alpha^{2} C_{\delta}^{2}<1 / 2$, where $C_{\delta}$ is given in Lemma 6.1. From (6.1) and (6.6), we can find a constant $c>0$ such that

$$
\begin{aligned}
0 \geq & \int_{\Omega}\left\{W_{\mathbf{h}}(x)-\alpha^{2}\left|\nabla \zeta_{\mathbf{h}}\right|^{2}-\lambda_{\varepsilon}+o\left(\varepsilon^{2 / 3}\right)\right\}\left|\eta \psi^{\varepsilon}\right|^{2} d x \\
\geq & \int_{\Omega\left(2 \varepsilon^{1 / 4}\right)}\left\{\lambda\left(\varphi_{\mathbf{h}}(x)\right)-\alpha^{2} C_{\delta}^{2}\left[\lambda\left(\varphi_{\mathbf{h}}(x)\right)-\beta_{0}\right]-\beta_{0}-(c+d) \varepsilon^{1 / 2}\right\}\left|\eta \psi^{\varepsilon}\right|^{2} d x \\
\geq & \frac{1}{2} \int_{\Omega\left(2 \varepsilon^{1 / 4}\right)}\left\{\lambda\left(\varphi_{\mathbf{h}}(x)\right)-\beta_{0}-2(c+d) \varepsilon^{1 / 2}\right\}\left|\eta \psi^{\varepsilon}\right|^{2} d x \\
\geq & \left(\frac{l}{2}-c-d\right) \varepsilon^{1 / 2} \int_{\Omega\left(2 \varepsilon^{1 / 4}\right) \cap\left\{\lambda\left(\varphi_{\mathbf{h}}(x)\right) \geq \beta_{0}+l \varepsilon^{1 / 2}\right\}}\left|\eta \psi^{\varepsilon}\right|^{2} d x \\
& -\left[\frac{\beta_{0}}{2}+(c+d) \varepsilon^{1 / 2}\right] \int_{\Omega\left(2 \varepsilon^{1 / 4}\right) \cap\left\{\lambda\left(\varphi_{\mathbf{h}}(x)\right)<\beta_{0}+l \varepsilon^{1 / 2}\right\}}\left|\eta \psi^{\varepsilon}\right|^{2} d x,
\end{aligned}
$$

where $l$ is chosen such that $l>2(c+d)$. So, for some $L_{1}>0$,

$$
\begin{aligned}
& \int_{\Omega\left(2 \varepsilon^{1 / 4}\right) \cap\left\{\lambda\left(\varphi_{\mathbf{h}}(x)\right) \geq \beta_{0}+l \varepsilon^{1 / 2}\right\}} \exp \left(2 \alpha \varepsilon^{-1} \zeta_{\mathbf{h}}(x)\right)\left|\psi^{\varepsilon}\right|^{2} d x \\
\leq & L_{1} \varepsilon^{-1 / 2} \int_{\Omega\left(2 \varepsilon^{1 / 4}\right) \cap\left\{\lambda\left(\varphi_{\mathbf{h}}(x)\right)<\beta_{0}+l \varepsilon^{1 / 2}\right\}} \exp \left(2 \alpha \varepsilon^{-1} \zeta_{\mathbf{h}}(x)\right)\left|\psi^{\varepsilon}\right|^{2} d x .
\end{aligned}
$$

In both sides we add

$$
\int_{\Omega\left(2 \varepsilon^{1 / 4}\right) \cap\left\{\lambda\left(\varphi_{\mathbf{h}}(x)\right)<\beta_{0}+l \varepsilon^{1 / 2}\right\}} \exp \left(2 \alpha \varepsilon^{-1} \zeta_{\mathbf{h}}(x)\right)\left|\psi^{\varepsilon}\right|^{2} d x
$$

to get

$$
\begin{aligned}
& \int_{\Omega\left(2 \varepsilon^{1 / 4}\right)} \exp \left(2 \alpha \varepsilon^{-1} \zeta_{\mathbf{h}}(x)\right)\left|\psi^{\varepsilon}\right|^{2} d x \\
\leq & \left(L_{1} \varepsilon^{-1 / 2}+1\right) \int_{\Omega\left(2 \varepsilon^{1 / 4}\right) \cap\left\{\lambda\left(\varphi_{\mathbf{h}}(x)\right)<\beta_{0}+l \varepsilon^{1 / 2}\right\}} \exp \left(2 \alpha \varepsilon^{-1} \zeta_{\mathbf{h}}(x)\right)\left|\psi^{\varepsilon}\right|^{2} d x .
\end{aligned}
$$

From Lemma 2.3 (conclusion (3)), on the set

$$
\Omega\left(2 \varepsilon^{1 / 4}\right) \cap\left\{\lambda\left(\varphi_{\mathbf{h}}(x)\right)<\beta_{0}+l \varepsilon^{1 / 2}\right\}
$$

we have

$$
\left|\varphi_{\mathbf{h}}(x)-\frac{\pi}{2}\right| \leq l^{1 / 2}\left(1-\beta_{0}\right)^{-1 / 2} \varepsilon^{1 / 4}+O\left(\varepsilon^{3 / 4}\right) .
$$

Then from Lemma 6.1 (conclusion (4)),

$$
\begin{aligned}
\zeta_{\mathbf{h}}(x) & =\zeta_{0}\left(\varphi_{\mathbf{h}}(x)\right) \leq \frac{2}{3}\left[l^{1 / 2}\left(1-\beta_{0}\right)^{-1 / 2} \varepsilon^{1 / 4}+O\left(\varepsilon^{3 / 4}\right)\right]^{3 / 2}+O\left(\varepsilon^{5 / 4}\right) \\
& =\frac{2}{3} l^{3 / 4}\left(1-\beta_{0}\right)^{-3 / 4} \varepsilon^{3 / 8}+O\left(\varepsilon^{7 / 8}\right) \leq c(l) \varepsilon^{3 / 8},
\end{aligned}
$$


where $c(l)$ is independent of $\varepsilon$. So, for some constant $L_{2}$,

$$
\begin{aligned}
& \int_{\Omega\left(2 \varepsilon^{1 / 4}\right)} \exp \left(2 \alpha \varepsilon^{-1} \zeta_{\mathbf{h}}(x)\right)\left|\psi^{\varepsilon}\right|^{2} d x \\
& \quad \leq L_{2} \varepsilon^{-1 / 2} \int_{\Omega\left(2 \varepsilon^{1 / 4}\right) \cap\left\{\lambda\left(\varphi_{\mathbf{h}}(x)\right)<\beta_{0}+l \varepsilon^{1 / 2}\right\}} \exp \left(2 \alpha \varepsilon^{-1} \zeta_{\mathbf{h}}(x)\right)\left|\psi^{\varepsilon}\right|^{2} d x \\
& \quad \leq L_{2} \varepsilon^{-1 / 2} \int_{\Omega\left(2 \varepsilon^{1 / 4}\right) \cap\left\{\lambda\left(\varphi_{\mathbf{h}}(x)\right)<\beta_{0}+l \varepsilon^{1 / 2}\right\}} \exp \left(2 \alpha \varepsilon^{-1} c(l) \varepsilon^{3 / 8}\right)\left|\psi^{\varepsilon}\right|^{2} d x .
\end{aligned}
$$

Thus

$$
\begin{gathered}
\int_{\Omega\left(2 \varepsilon^{1 / 4}\right) \cap\left\{\lambda\left(\varphi_{\mathbf{h}}(x)\right) \geq \beta_{0}+l \varepsilon^{1 / 2}\right\}} \exp \left(2 \alpha \varepsilon^{-1}\left[\zeta_{\mathbf{h}}(x)-c(l) \varepsilon^{3 / 8}\right]\right)\left|\psi^{\varepsilon}\right|^{2} d x \\
\quad \leq L_{2} \varepsilon^{-1 / 2} \int_{\Omega\left(2 \varepsilon^{1 / 4}\right) \cap\left\{\lambda\left(\varphi_{\mathbf{h}}(x)\right)<\beta_{0}+l \varepsilon^{1 / 2}\right\}}\left|\psi^{\varepsilon}\right|^{2} d x .
\end{gathered}
$$

Then we use (5.5) to control the integrals on $\Omega \backslash \Omega(\varepsilon)$ and find

$$
\int_{\Omega} \exp \left(2 \alpha \varepsilon^{-1}\left[\zeta_{\mathbf{h}}(x)-c(l) \varepsilon^{3 / 8}\right]\right)\left|\psi^{\varepsilon}\right|^{2} d x \leq L_{3} \varepsilon^{-1 / 2} \int_{\Omega(\varepsilon)}\left|\psi^{\varepsilon}\right|^{2} d x,
$$

where $L_{3}>0$. Now (6.2) is proved.

If $x \in \Omega(\delta)$ and $\left|\varphi_{\mathbf{h}}(x)-\frac{\pi}{2}\right| \geq c \kappa^{-3 / 16}$, from Lemma 6.1 (conclusion (4)) we have

$$
\begin{aligned}
\zeta_{\mathbf{h}}(x) & =\zeta_{0}\left(\varphi_{\mathbf{h}}(x)\right) \geq \frac{1}{2} \sqrt{1-\beta_{0}}\left(\varphi_{\mathbf{h}}(x)-\frac{\pi}{2}\right)^{2}+O\left(\left(\varphi_{\mathbf{h}}(x)-\frac{\pi}{2}\right)^{4}\right) \\
& \geq \frac{c^{2}}{2} \sqrt{1-\beta_{0}} \kappa^{-3 / 8}+O\left(\kappa^{3 / 4}\right) .
\end{aligned}
$$

Let the constant $c$ be chosen sufficiently large. Then we can find a positive constant $m$, such that on the set

$$
\Omega(\delta) \cap\left\{\left|\varphi_{\mathbf{h}}(x)-\frac{\pi}{2}\right| \geq c \kappa^{-3 / 16}\right\}
$$

we have

$$
\alpha_{1} \kappa\left[\zeta_{\mathbf{h}}(x)-c_{1} \kappa^{-5 / 8}\right] \geq m \kappa^{5 / 8}
$$

for all large $\kappa$, where $\alpha_{1}$ and $c_{1}$ are the constants in (6.2). From (6.2) we have

$$
\int_{\Omega(\delta) \cap\left\{\left|\varphi_{\mathbf{h}}(x)-\frac{\pi}{2}\right| \geq c \kappa^{-3 / 16}\right\}}|\psi|^{2} d x \leq M_{1} \kappa^{1 / 2} \exp \left(-m \kappa^{-5 / 8}\right) \int_{\Omega\left(\kappa^{-1}\right)}|\psi|^{2} d x .
$$

Now we choose $0<\gamma_{1}<m$ such that

$$
\kappa^{1 / 2} \exp \left(-m \kappa^{-5 / 8}\right) \leq \exp \left(-\gamma_{1} \kappa^{-5 / 8}\right)
$$

holds for all large $\kappa$. Then (6.3) follows.

Step 2. In case (2) we have $\varepsilon=\sqrt{b} \kappa^{-1}$ and $\lambda_{\varepsilon}=b$. We choose $\zeta=\zeta_{\mathbf{h}, b}$ and choose $\alpha$ such that $\alpha^{2} C_{\delta, b}^{2} \leq 1 / 2$, where $C_{\delta, b}$ is given in Lemma 6.2. From (6.6) 
and Lemma 6.2 (conclusion (3)),

$$
\begin{aligned}
0 \geq & \int_{\Omega}\left\{W_{\mathbf{h}}(x)-\alpha^{2}\left|\nabla \zeta_{\mathbf{h}, b}\right|^{2}-b+o\left(\varepsilon^{2 / 3}\right)\right\}\left|\eta \psi^{\varepsilon}\right|^{2} d x \\
\geq & \int_{\Omega\left(2 \varepsilon^{1 / 4}\right)}\left\{\lambda\left(\varphi_{\mathbf{h}}(x)\right)-\alpha^{2} C_{\delta, b}^{2}\left[\lambda\left(\varphi_{\mathbf{h}}(x)\right)-b\right]_{+}-b-c \varepsilon^{1 / 2}\right\}\left|\eta \psi^{\varepsilon}\right|^{2} d x \\
\geq & \int_{\Omega\left(2 \varepsilon^{1 / 4}\right)}\left\{\lambda\left(\varphi_{\mathbf{h}}(x)\right)-b-\frac{1}{2}\left[\lambda\left(\varphi_{\mathbf{h}}(x)\right)-b\right]_{+}-c \varepsilon^{1 / 2}\right\}\left|\eta \psi^{\varepsilon}\right|^{2} d x \\
\geq & \left(\frac{l}{2}-c\right) \varepsilon^{1 / 2} \int_{\Omega\left(2 \varepsilon^{1 / 4}\right) \cap\left\{\lambda\left(\varphi_{\mathbf{h}}(x)\right) \geq b+l \varepsilon^{1 / 2}\right\}}\left|\eta \psi^{\varepsilon}\right|^{2} d x \\
& -\left(b+c \varepsilon^{1 / 2}\right) \int_{\Omega\left(2 \varepsilon^{1 / 4}\right) \cap\left\{\lambda\left(\varphi_{\mathbf{h}}(x)\right)<b+l \varepsilon^{1 / 2}\right\}}\left|\eta \psi^{\varepsilon}\right|^{2} d x,
\end{aligned}
$$

where $l$ is chosen such that $l>2 c$. Then we argue as in Step 1 and use Lemma 6.2 (conclusion (4)) to find

$$
\begin{aligned}
& \int_{\Omega\left(2 \varepsilon^{1 / 4}\right)} \exp \left(2 \alpha \varepsilon^{-1}\left[\zeta_{\mathbf{h}, b}(x)-c_{l}(\varepsilon) \varepsilon^{1 / 4}\right]\right)\left|\psi^{\varepsilon}\right|^{2} d x \\
\leq & L_{4} \varepsilon^{-1 / 2} \int_{\Omega\left(2 \varepsilon^{1 / 4}\right) \cap\left\{\lambda\left(\varphi_{\mathbf{h}}(x)\right)<b+l \varepsilon^{1 / 2}\right\}}\left|\psi^{\varepsilon}\right|^{2} d x,
\end{aligned}
$$

and hence

$$
\int_{\Omega} \exp \left(2 \alpha \varepsilon^{-1}\left[\zeta_{\mathbf{h}, b}(x)-c_{l}(\varepsilon) \varepsilon^{1 / 4}\right]\right)\left|\psi^{\varepsilon}\right|^{2} d x \leq L_{5} \varepsilon^{-1 / 2} \int_{\Omega(\varepsilon)}\left|\psi^{\varepsilon}\right|^{2} d x,
$$

where $L_{4}$ and $L_{5}$ are positive constants. So (6.4) is true.

Let $c_{2}$ be the constant in (6.4). For large $\kappa$ we define

$$
\eta(\kappa)=\sup \left\{\eta>0: \int_{\lambda^{-1}(b)-\eta}^{\lambda^{-1}(b)}[\lambda(\vartheta)-b]^{1 / 2} d \vartheta \leq 2 c_{2} \kappa^{-1 / 4}\right\} .
$$

Obviously, $\lim _{\kappa \rightarrow \infty} \eta(\kappa)=0$. If $0 \leq \varphi \leq \lambda^{-1}(b)-\eta(\kappa)$, then

$$
\zeta_{b}(\varphi) \geq \int_{\lambda^{-1}(b)-\eta(\kappa)}^{\lambda^{-1}(b)}[\lambda(\vartheta)-b]^{1 / 2} d \vartheta \geq 2 c_{2} \kappa^{-1 / 4} .
$$

If $\pi-\lambda^{-1}(b)+\eta(\kappa) \leq \varphi \leq \pi$, then $0 \leq \pi-\varphi \leq \lambda^{-1}(b)-\eta(\kappa)$, and by the definition of $\zeta_{b}$ we have

$$
\zeta_{b}(\varphi)=\zeta_{b}(\pi-\varphi) \geq 2 c_{2} \kappa^{-1 / 4}
$$

Thus we can use (6.4) and argue as in the last part of Step 1 to derive (6.5).

Proof of Theorem 2. It is a corollary of Theorem 6.3.

Appendix A. Computational details for equation (2.8)

Consider equation (2.8). Using the new variables $\xi=\left(\xi_{1}, \xi_{2}\right)$ defined in (2.10), we write equation (2.8) as follows:

$$
-\nabla_{\mathbf{Q}}^{2} \psi_{1}=\mu_{p} \psi_{1}+f(\xi) \psi_{1}, \quad \xi \in \mathbb{R}^{2},
$$


where the vector field $\mathbf{Q}(\xi)=\left(Q_{1}(\xi), Q_{2}(\xi)\right)$ and the function $f(\xi)$ are given by

$$
\begin{aligned}
& Q_{1}(\xi)=\frac{1}{\varepsilon_{1}}\left[\frac{1}{\sqrt{c_{11}}} f_{1}(y)+\frac{1}{\sqrt{c_{22}}} f_{2}(y)\right], \\
& Q_{2}(\xi)=\frac{1}{\varepsilon_{2}}\left[\frac{1}{\sqrt{c_{11}}} f_{1}(y)-\frac{1}{\sqrt{c_{22}}} f_{2}(y)\right], \\
& f(\xi)=f_{1}^{2}(y)+f_{2}^{2}(y)-f_{0}(y)+i \operatorname{div} \mathbf{Q},
\end{aligned}
$$

where $f_{0}, f_{1}$ and $f_{2}$ are given in (2.9). We compute $Q_{1}(\xi)$ and $Q_{2}(\xi)$ to get

$$
\begin{aligned}
& Q_{1}(\xi)=\alpha_{11} \xi_{1}^{2}+2 \alpha_{12} \xi_{1} \xi_{2}+\alpha_{22} \xi_{2}^{2}, \\
& Q_{2}(\xi)=\beta_{11} \xi_{1}^{2}+2 \beta_{12} \xi_{1} \xi_{2}+\beta_{22} \xi_{2}^{2},
\end{aligned}
$$

where

$$
\begin{aligned}
& \alpha_{11}=\frac{\varepsilon_{1}}{4}\left[-\left(\sqrt{c_{11}} c_{22}+c_{12} \sqrt{c_{22}}\right) a_{2}^{(1)}+\left(c_{11} \sqrt{c_{22}}+\sqrt{c_{11}} c_{12}\right) a_{1}^{(2)}\right], \\
& \alpha_{12}=\frac{\varepsilon_{2}}{4}\left[\left(\sqrt{c_{11}} c_{22}+c_{12} \sqrt{c_{22}}\right) a_{2}^{(1)}+\left(c_{11} \sqrt{c_{22}}+\sqrt{c_{11}} c_{12}\right) a_{1}^{(2)}\right], \\
& \alpha_{22}=\frac{\varepsilon_{2}^{2}}{4 \varepsilon_{1}}\left[-\left(\sqrt{c_{11}} c_{22}+c_{12} \sqrt{c_{22}}\right) a_{2}^{(1)}+\left(c_{11} \sqrt{c_{22}}+\sqrt{c_{11}} c_{12}\right) a_{1}^{(2)}\right], \\
& \beta_{11}=\frac{\varepsilon_{1}^{2}}{4 \varepsilon_{2}}\left[\left(-\sqrt{c_{11}} c_{22}+c_{12} \sqrt{c_{22}}\right) a_{2}^{(1)}+\left(-c_{11} \sqrt{c_{22}}+\sqrt{c_{11}} c_{12}\right) a_{1}^{(2)}\right], \\
& \beta_{12}=\frac{\varepsilon_{1}}{4}\left[\left(\sqrt{c_{11}} c_{22}-c_{12} \sqrt{c_{22}}\right) a_{2}^{(1)}+\left(-c_{11} \sqrt{c_{22}}+\sqrt{c_{11}} c_{12}\right) a_{1}^{(2)}\right], \\
& \beta_{22}=\frac{\varepsilon_{2}}{4}\left[\left(-\sqrt{c_{11}} c_{22}+c_{12} \sqrt{c_{22}}\right) a_{2}^{(1)}+\left(-c_{11} \sqrt{c_{22}}+\sqrt{c_{11}} c_{12}\right) a_{1}^{(2)}\right] .
\end{aligned}
$$

Computations show that

$$
f(\xi) \equiv 0 .
$$

Hence we can write the equation for $\psi_{1}(\xi)$ in the following form:

$$
-\nabla_{\mathbf{Q}}^{2} \psi_{1}=\mu_{2} \psi_{1} \quad \text { in } \mathbb{R}^{2},
$$

which is exactly (2.11). We can make a gauge transformation to rewrite (2.11) in a form more convenient to us. Let us choose

$$
\eta(\xi)=\frac{1}{3}\left(\alpha_{11}-\alpha_{22}+\beta_{12}\right) \xi_{1}^{2}+\alpha_{12} \xi_{1}^{2} \xi_{2}+\beta_{12} \xi_{1} \xi_{2}^{2}+\frac{1}{3}\left(\alpha_{12}-\beta_{11}+\beta_{22}\right) \xi_{2}^{3},
$$

and let

$$
\begin{aligned}
& \gamma_{1}=\beta_{12}-\alpha_{22}=2 \frac{c_{11} c_{22}-\left(c_{12}\right)^{2}}{\varepsilon_{1} \sqrt{c_{11} c_{22}}}\left(\sqrt{c_{22}} a_{2}^{(1)}-\sqrt{c_{11}} a_{1}^{(2)}\right), \\
& \gamma_{2}=\alpha_{12}-\beta_{11}=2 \frac{c_{11} c_{22}-\left(c_{12}\right)^{2}}{\varepsilon_{2} \sqrt{c_{11} c_{22}}}\left(\sqrt{c_{22}} a_{2}^{(1)}+\sqrt{c_{11}} a_{1}^{(2)}\right) ;
\end{aligned}
$$

see (2.12). Then

$$
\mathbf{Q}(\xi)=-\frac{|\xi|^{2}}{2}\left(\gamma_{1}, \gamma_{2}\right)+\nabla \eta(\xi)
$$

Let

$$
\begin{aligned}
& \rho=\left(\gamma_{1}^{2}+\gamma_{2}^{2}\right)^{1 / 2}, \quad \gamma_{1}=\rho \cos \vartheta, \quad \gamma_{2}=\rho \sin \vartheta \\
& \mathbf{A}_{\vartheta}(\xi)=-\frac{|\xi|^{2}}{2}(\cos \vartheta, \sin \vartheta) .
\end{aligned}
$$


Then we have

$$
\mathbf{Q}=\rho \mathbf{A}_{\vartheta}(\xi)+\nabla \eta
$$

We introduce $\psi_{2}(\xi)=\psi_{1}(\xi) e^{-i \eta}$, and derive an equation for $\psi_{2}$ from (2.11):

$$
-\nabla_{\rho \mathbf{A}_{\vartheta}}^{2} \psi_{2}=\mu_{p} \psi_{2} \quad \text { in } \mathbb{R}^{2} .
$$

We make further change of variables as in (2.13), and let

$$
\varphi(x)=\psi_{2}(\xi) \exp \left(\frac{i}{6} x_{1}^{3}\right), \quad \lambda_{0}=\mu_{p} \rho^{-2 / 3} .
$$

Then $\varphi(x)$ satisfies (2.14).

\section{Appendix B. Proof of Lemma 3.1}

We keep the notation in Section 3. In particular, let $X_{0} \in \partial \Omega$ be fixed and $(y, z)$ be the new variables introduced there. We first consider the following problem: Given a vector field $\mathbf{R}$ satisfying $\operatorname{div} \mathbf{R}=0$, find a vector field $\mathbf{A}$ such that

$$
\operatorname{curl} \mathbf{A}=\mathbf{R} \quad \text { in } \Omega(\delta) .
$$

To solve this question, we write

$$
\mathbf{R}=\sum_{j=1}^{3} G^{j j} R_{j} \partial_{j} \mathcal{F}, \quad \text { where } \quad R_{j}=\mathbf{R} \cdot \partial_{j} \mathcal{F}
$$

Since $\operatorname{div} \mathbf{R}=0$, we have

$$
\sum_{j=1}^{3} \partial_{j}\left(\sqrt{G} G^{j j} R_{j}\right)=0 .
$$

(B.1) has a solution

$$
\mathbf{A}=\sum_{j=1}^{3} G^{j j} a_{j} \partial_{j} \mathcal{F}
$$

where $a_{j}$ are given by

$$
\begin{aligned}
& a_{1}(y, z)=\int_{0}^{z} \sqrt{G(y, t)} G^{22}(y, t) R_{2}(y, t) d t \\
& a_{2}(y, z)=-\int_{0}^{z} \sqrt{G(y, t)} G^{11}(y, t) R_{1}(y, t) d t+p_{2}(y), \\
& a_{3}(y, z)=0,
\end{aligned}
$$

where

$$
p_{2}(y)=\int_{0}^{y_{1}} \sqrt{G\left(s, y_{2}, 0\right)} R_{3}\left(s, y_{2}, 0\right) d s .
$$

Now, given a unit vector $\mathbf{h}$, we can use (B.3) to find a vector field $\mathbf{A}_{\mathbf{h}}$ that satisfies

$$
\operatorname{curl} \mathbf{A}=\mathbf{h} \quad \text { in } \Omega(\delta) .
$$


Write

$$
\mathbf{h}=\sum_{j=1}^{3} G^{j j} R_{j} \partial_{j} \mathcal{F}
$$

where

$$
\begin{aligned}
& R_{1}=\left[1-\kappa_{1}(y) z\right] \mathbf{h} \cdot \mathbf{r}_{1}(y), \\
& R_{2}=\left[1-\kappa_{2}(y) z\right] \mathbf{h} \cdot \mathbf{r}_{2}(y), \\
& R_{3}=\mathbf{h} \cdot \mathbf{n}(y) .
\end{aligned}
$$

Then the solution of (B.4) is

$$
\mathbf{A}=\mathbf{A}_{\mathbf{h}}+\nabla \chi,
$$

where $\chi$ is an arbitrary smooth function, and

$$
\mathbf{A}_{\mathbf{h}}=\sum_{j=1}^{3} G^{j j} a_{j} \partial_{j} \mathcal{F},
$$

where

$$
\begin{aligned}
& a_{1}(y, z)=\sqrt{\frac{g_{11}(y)}{g_{22}(y)}}\left[z-\frac{1}{2} \kappa_{2}(y) z^{2}\right] \mathbf{h} \cdot \mathbf{r}_{2}(y), \\
& a_{2}(y, z)=-\sqrt{\frac{g_{22}(y)}{g_{11}(y)}}\left[z-\frac{1}{2} \kappa_{1}(y) z^{2}\right] \mathbf{h} \cdot r_{1}(y) \\
& +\int_{0}^{y_{1}} \sqrt{g\left(s, y_{2}\right)} \mathbf{h} \cdot \mathbf{n}\left(s, y_{2}\right) d s, \\
& a_{3}(y, z)=0 .
\end{aligned}
$$

In the following we look for a solution to (B.4) which has a simple expansion in a neighborhood of $X_{0}$. Choose $\mathbf{e}_{j}$ as in (3.2). Then (3.3) holds. For the unit vector h, we have

$$
\begin{aligned}
& \mathbf{h} \cdot \mathbf{r}_{1}(y)=h_{1}+\left(h_{1} \Gamma_{1 j}^{1}+h_{2} \Gamma_{1 j}^{2}\right) y_{j}+h_{3} \Omega_{11} y_{1}+O\left(|y|^{2}\right), \\
& \mathbf{h} \cdot \mathbf{r}_{2}(y)=h_{2}+\left(h_{1} \Gamma_{2 j}^{1}+h_{2} \Gamma_{2 j}^{2}\right) y_{j}+h_{3} \Omega_{22} y_{2}+O\left(|y|^{2}\right), \\
& \mathbf{h} \cdot \mathbf{n}(y)=h_{3}-h_{j} g^{j j} \Omega_{j j} y_{j}+O\left(|y|^{2}\right) .
\end{aligned}
$$

From (3.4), (3.6), (B.5) and (B.6) we get

$$
\begin{aligned}
& a_{1}(y, z)=h_{2} \sqrt{\frac{g_{11}}{g_{22}}} z+a_{13}^{(1)} y_{1} z+a_{23}^{(1)} y_{2} z+a_{33}^{(1)} z^{2}+O\left(|y|^{3}+|z|^{3}\right) \\
& \begin{aligned}
a_{2}(y, z)= & h_{3} \sqrt{g} y_{1}-h_{1} \sqrt{\frac{g_{22}}{g_{11}}} z+a_{1}^{(2)} y_{1}^{2}+2 a_{2}^{(1)} y_{1} y_{2}+a_{13}^{(2)} y_{1} z \\
& \quad+a_{23}^{(2)} y_{2} z+a_{33}^{(2)} z^{2}+O\left(|y|^{3}+|z|^{3}\right)
\end{aligned} \\
& a_{3}(y, z)=0
\end{aligned}
$$


where

$$
\begin{aligned}
& a_{2}^{(1)}=\sqrt{g}\left[\frac{h_{3}}{4}\left(\frac{g_{11,2}}{g_{11}}+\frac{g_{22,2}}{g_{22}}\right)-\frac{h_{2}}{2} \kappa_{2}\right], \\
& a_{13}^{(1)}=\sqrt{\frac{g_{11}}{g_{22}}}\left[\frac{h_{2}}{2}\left(\frac{g_{11,1}}{g_{11}}-\frac{g_{22,1}}{g_{22}}\right)+h_{1} \Gamma_{21}^{1}+h_{2} \Gamma_{21}^{2}\right], \\
& a_{23}^{(1)}=\sqrt{\frac{g_{11}}{g_{22}}}\left[\frac{h_{2}}{2}\left(\frac{g_{11,2}}{g_{11}}-\frac{g_{22,2}}{g_{22}}\right)+h_{1} \Gamma_{22}^{1}+h_{2} \Gamma_{22}^{2}+h_{3} \Omega_{22}\right], \\
& a_{33}^{(1)}=-\frac{h_{2}}{2} \sqrt{\frac{g_{11}}{g_{22}}} \kappa_{1}, \\
& a_{1}^{(2)}=\sqrt{g}\left[\frac{h_{3}}{4}\left(\frac{g_{11,1}}{g_{11}}+\frac{g_{22,1}}{g_{22}}\right)-\frac{h_{1}}{2} \kappa_{1}\right], \\
& a_{13}^{(2)}=\sqrt{\frac{g_{22}}{g_{11}}}\left[\frac{h_{1}}{2}\left(\frac{g_{11,1}}{g_{11}}-\frac{g_{22,1}}{g_{22}}\right)-h_{1} \Gamma_{11}^{1}-h_{2} \Gamma_{11}^{2}-h_{3} \Omega_{11}\right], \\
& a_{23}^{(2)}=\sqrt{\frac{g_{22}}{g_{11}}}\left[\frac{h_{1}}{2}\left(\frac{g_{11,2}}{g_{11}}-\frac{g_{22,2}}{g_{22}}\right)-h_{1} \Gamma_{12}^{1}-h_{2} \Gamma_{12}^{2}\right], \\
& a_{33}^{(2)}=\frac{h_{1}}{2} \sqrt{\frac{g_{22}}{g_{11}}} \kappa_{2} .
\end{aligned}
$$

From (B.2) we have

$$
\begin{aligned}
& \partial_{1}\left\{\sqrt{\frac{g_{22}(y)}{g_{11}(y)}}\left[1-\kappa_{2}(y) z\right] \mathbf{h} \cdot \mathbf{r}_{1}(y)\right\}+\partial_{2}\left\{\sqrt{\frac{g_{11}(y)}{g_{22}(y)}}\left[1-\kappa_{1}(y) z\right] \mathbf{h} \cdot \mathbf{r}_{2}(y)\right\} \\
& +\partial_{z}\left\{\sqrt{g(y)}\left[1-\kappa_{1}(y) z\right]\left[1-\kappa_{2}(y) z\right] \mathbf{h} \cdot \mathbf{n}(y)\right\}=0 .
\end{aligned}
$$

Checking the Taylor expansion of this equality we find that

$$
a_{23}^{(1)}=a_{13}^{(2)}+h_{3}\left(\kappa_{1}+\kappa_{2}\right) \sqrt{g} .
$$

Now we fix the point $X_{0} \in(\partial \Omega)_{\mathbf{h}}$, namely, $\mathbf{h}$ is orthogonal to $\mathbf{n}$ at $X_{0}$. Thus

$$
\begin{aligned}
& a_{2}^{(1)}=-\frac{h_{2}}{2} \sqrt{g} \kappa_{2}, \quad a_{1}^{(2)}=-\frac{h_{1}}{2} \sqrt{g} \kappa_{1}, \quad a_{23}^{(1)}=a_{13}^{(2)}, \\
& h_{3}=0, \quad \frac{h_{1}^{2}}{g_{11}}+\frac{h_{2}^{2}}{g_{22}}=1 .
\end{aligned}
$$

Recall that $y_{1}, y_{2}$ were chosen such that the $y_{1}$ - and $y_{2}$-curves are the lines of curvatures on $\partial \Omega$. Using (B.10) and the relation between the Christoffel symbols and $g_{i i, j}$ we find

$$
\begin{aligned}
& a_{13}^{(1)}=\frac{1}{2 \sqrt{g}}\left(h_{1} g_{11,2}+h_{2} g_{11,1}\right), \\
& a_{23}^{(1)}=\frac{1}{2 \sqrt{g}}\left(-h_{1} g_{22,1}+h_{2} g_{11,2}\right), \\
& a_{23}^{(2)}=-\frac{1}{2 \sqrt{g}}\left(h_{1} g_{22,2}+h_{2} g_{22,1}\right) .
\end{aligned}
$$

From (B.8), (B.10), (B.11) we get (3.10). 
To simplify our computations we modify $\mathbf{A}_{\mathbf{h}}$ by a gauge transformation. Let $\xi_{0}$ be a real number, the value of which is to be determined later. Set

$$
\chi(y)=a_{2}^{(1)} y_{1} y_{2}^{2}-\xi_{0}\left\{h_{2} \sqrt{\frac{g_{11}}{g_{22}}} y_{1}-h_{1} \sqrt{\frac{g_{22}}{g_{11}}} y_{2}+\frac{1}{2} a_{13}^{(1)} y_{1}^{2}+a_{23}^{(1)} y_{1} y_{2}+\frac{1}{2} a_{23}^{(2)} y_{2}^{2}\right\},
$$

and let

$$
\begin{aligned}
& \mathbf{F}_{\mathbf{h}}=\mathbf{A}_{\mathbf{h}}-\nabla \chi=\sum_{j=1}^{3} G^{j j} \hat{a}_{j} \partial_{j} \mathcal{F}, \\
& \text { where } \quad \hat{a}_{j}(y, z)=a_{j}(y, z)-\partial_{j} \chi(y), \quad j=1,2,3 .
\end{aligned}
$$

Using (B.10) we find that $\hat{a}_{j}(y, z)$ satisfy (3.9). Now Lemma 3.1 is proved.

\section{Appendix C. Formal expansion of the lowest eigenvalue}

In this section we look for a formal solution of (3.13). We shall keep the notation in Section 3 and Appendix B. Recall that we fixed a point $X_{0} \in(\partial \Omega)_{\mathbf{h}}$. Write

$$
\mu=\mu_{0} \varepsilon^{-2}+\mu_{1} \varepsilon^{-5 / 3}+\mu_{2} \varepsilon^{-4 / 3}+O\left(\varepsilon^{-1}\right) .
$$

Using (3.5), (3.14) and (B.10), we can expand (3.13) as follows:

(C.1)

$$
\begin{aligned}
& \partial_{z z} \phi-\left(z+z_{0}\right)^{2} \phi+\mu_{0} \phi \\
& +\varepsilon^{1 / 3}\left\{\frac{2\left(z+z_{0}\right)}{\sqrt{g}}\left[-i h_{2} \partial_{1} \phi+i h_{1} \partial_{2} \phi+\left(a_{1}^{(2)} h_{1} y_{1}^{2}+a_{2}^{(1)} h_{2} y_{2}^{2}\right) \phi\right]+\mu_{1} \phi\right\} \\
& +\varepsilon^{2 / 3}\left\{\frac{\partial_{11} \phi}{g_{11}}+\frac{\partial_{22} \phi}{g_{22}}+2 i \frac{a_{2}^{(1)}}{g_{11}} y_{2}^{2} \partial_{1} \phi-2 i \frac{a_{1}^{(2)}}{g_{22}} y_{1}^{2} \partial_{2} \phi-\left[\frac{\left(a_{1}^{(2)}\right)^{2}}{g_{22}} y_{1}^{4}+\frac{\left(a_{2}^{(1)}\right)^{2}}{g_{11}} y_{2}^{4}\right] \phi\right. \\
& +\left(z+z_{0}\right)^{2}\left[\frac{2 h_{1}}{\sqrt{g}}\left(a_{13}^{(2)} y_{1}+a_{23}^{(2)} y_{2}\right)-\frac{2 h_{2}}{\sqrt{g}}\left(a_{13}^{(1)} y_{1}+a_{23}^{(1)} y_{2}\right)\right. \\
& \left.\left.\quad+\frac{1}{g}\left(h_{2}^{2} g_{11, j}+h_{1}^{2} g_{22, j}\right) y_{j}\right] \phi+\mu_{2} \phi\right\} \\
& +O(\varepsilon)=0,
\end{aligned}
$$

where $\partial_{j}=\partial_{y_{j}}, j=1,2$. Using (B.8), (B.10) and (B.11) we find that

$$
\begin{aligned}
& h_{1}^{2} \frac{g_{22,1}}{\sqrt{g}}+h_{2}^{2} \frac{g_{11,1}}{\sqrt{g}}+2 h_{1} a_{13}^{(2)}-2 h_{2} a_{13}^{(1)}=0, \\
& h_{1}^{2} \frac{g_{22,2}}{\sqrt{g}}+h_{2}^{2} \frac{g_{11,2}}{\sqrt{g}}+2 h_{1} a_{23}^{(2)}-2 h_{2} a_{23}^{(1)}=0 .
\end{aligned}
$$

Thus we can write the equation as follows:

$$
\begin{aligned}
& \partial_{z z} \phi-\left(z+z_{0}\right)^{2} \phi+\mu_{0} \phi \\
+ & \varepsilon^{1 / 3}\left\{\frac{2\left(z+z_{0}\right)}{\sqrt{g}}\left[-i h_{2} \partial_{1} \phi+i h_{1} \partial_{2} \phi+\left(a_{1}^{(2)} h_{1} y_{1}^{2}+a_{2}^{(1)} h_{2} y_{2}^{2}\right) \phi\right]+\mu_{1} \phi\right\} \\
+ & \varepsilon^{2 / 3}\left\{\frac{\partial_{11} \phi}{g_{11}}+\frac{\partial_{22} \phi}{g_{22}}+2 i \frac{a_{2}^{(1)}}{g_{11}} y_{2}^{2} \partial_{1} \phi-2 i \frac{a_{1}^{(2)}}{g_{22}} y_{1}^{2} \partial_{2} \phi\right. \\
& \left.\quad-\left[\frac{\left(a_{1}^{(2)}\right)^{2}}{g_{22}} y_{1}^{4}+\frac{\left(a_{2}^{(1)}\right)^{2}}{g_{11}} y_{2}^{4}\right] \phi+\mu_{2} \phi\right\} \\
+ & O(\varepsilon)=0 .
\end{aligned}
$$


We look for a solution in the form

$$
\phi=\phi_{0}+\varepsilon^{1 / 3} \phi_{1}+\varepsilon^{2 / 3} \phi_{2}+O(\varepsilon),
$$

where $\phi_{j}$ are functions defined in $\mathbb{R}_{+}^{3}$. Plugging it into (C.3) and checking the order 1 terms we find an equation for $\phi_{0}$ :

$$
\partial_{z z} \phi_{0}-\left(z+z_{0}\right)^{2} \phi+\mu_{0} \phi=0 \quad \text { in } \mathbb{R}_{+}^{3}, \quad \partial_{z} \phi_{0}=0 \quad \text { on } \partial \mathbb{R}_{+}^{3} .
$$

Using Lemma 2.1 we find that

$$
\mu_{0}=\beta_{0}, \quad \phi_{0}(y, z)=w(y) u(z),
$$

$z_{0}$ is the minimum point of $\beta(z)$ given in Lemma 2.1, and $u$ is the eigenfunction of (2.1) for $z_{0}$ and $\beta_{0}$.

Next, by checking the $O\left(\varepsilon^{1 / 3}\right)$ terms in (C.3), we find an equation for $\phi_{1}$ :

$$
\begin{aligned}
& \partial_{z z} \phi_{1}-\left(z+z_{0}\right)^{2} \phi_{1}+\beta_{0} \phi_{1} \\
& +\frac{2\left(z+z_{0}\right) u}{\sqrt{g}}\left[-i h_{2} \partial_{1} w+i h_{1} \partial_{2} w+\left(a_{1}^{(2)} h_{1} y_{1}^{2}+a_{2}^{(1)} h_{2} y_{2}^{2}\right) w\right]+\mu_{1} u w=0 .
\end{aligned}
$$

We fix $y$, multiply (C.4) by $u(z)$, integrate the equality in $z$ over $[0,+\infty)$, and use the first equality in $(2.2)$ to find that

$$
\mu_{1}=0 .
$$

Let us look for a solution $\phi_{1}$ of (C.4) in the form

$$
\phi_{1}=w_{1}(y) u_{1}(z) \text {. }
$$

Plugging it into (C.4) we find that $u_{1}$ satisfies (2.3), and

$$
w_{1}(y)=\frac{2}{\sqrt{g}}\left[i h_{2} \partial_{1} w-i h_{1} \partial_{2} w-\left(a_{1}^{(2)} h_{1} y_{1}^{2}+a_{2}^{(1)} h_{2} y_{2}^{2}\right) w\right] .
$$

Now we check the $O\left(\varepsilon^{2 / 3}\right)$ terms in (C.3) and find an equation for $\phi_{2}$ :

$$
\begin{gathered}
\partial_{z z} \phi_{2}-\left(z+z_{0}\right)^{2} \phi_{2}+\beta_{0} \phi_{2} \\
+\frac{2\left(z+z_{0}\right) u_{1}}{\sqrt{g}}\left[-i h_{2} \partial_{1} w_{1}+i h_{1} \partial_{2} w_{1}+\left(a_{1}^{(2)} h_{1} y_{1}^{2}+a_{2}^{(1)} h_{2} y_{2}^{2}\right) w_{1}\right] \\
+u\left\{\frac{\partial_{11} w}{g_{11}}+\frac{\partial_{22} w}{g_{22}}+2 i \frac{a_{2}^{(1)}}{g_{11}} y_{2}^{2} \partial_{1} w-2 i \frac{a_{1}^{(2)}}{g_{22}} y_{1}^{2} \partial_{2} w\right. \\
\left.-\left[\frac{\left(a_{1}^{(2)}\right)^{2}}{g_{22}} y_{1}^{4}+\frac{\left(a_{2}^{(1)}\right)^{2}}{g_{11}} y_{2}^{4}\right] w+\mu_{2} w\right\}=0 .
\end{gathered}
$$

Multiplying the equation by $u$ and integrating in $z$, and using (C.5) and (2.4), we find that, $w$ satisfies the following equality:

$$
c_{11} \partial_{11} w+2 c_{12} \partial_{12} w+c_{22} \partial_{22} w-2 i f_{1} \partial_{1} w-2 i f_{2} \partial_{2} w-f_{0} w+\mu_{2} w=0,
$$

where $c_{i j}$ and $f_{j}$ are given in (4.6). Computations show that

$$
c_{11} c_{22}-c_{12}^{2}=\frac{1-\alpha_{0}}{g}>0,
$$

where $\alpha_{0}$ is the number given in (2.4). Comparing (4.6) with (2.9), we see that the equation (C.6) is in the form of (2.8). From Proposition 2.5 we know that, the lowest eigenvalue of $(\mathrm{C} .6)$ is $\lambda_{0}\left(\gamma_{1}^{2}+\gamma_{2}^{2}\right)^{1 / 3}$, where $\lambda_{0}$ is the lowest eigenvalue of 
(2.14), and $\gamma_{1}$ and $\gamma_{2}$ are determined by (2.12). From the last equality in (B.10), we can write

$$
\begin{aligned}
& \cos \theta\left(X_{0}\right)=\frac{h_{1}}{\sqrt{g_{11}}}=\mathbf{h} \cdot \frac{\mathbf{r}_{1}(0,0)}{\sqrt{g_{11}(0,0)}}, \\
& \sin \theta\left(X_{0}\right)=\frac{h_{2}}{\sqrt{g_{22}}}=\mathbf{h} \cdot \frac{\mathbf{r}_{2}(0,0)}{\sqrt{g_{22}(0,0)}} .
\end{aligned}
$$

$\theta\left(X_{0}\right)$ is actually the angle between $\mathbf{h}$ and the principal direction $\mathbf{e}_{1}(0,0)$ at $X_{0}$. Then we compute

$$
\begin{aligned}
& \gamma_{1}^{2}+\gamma_{2}^{2}=4\left(c_{11} c_{22}-c_{12}^{2}\right)\left[c_{11}\left(a_{1}^{(2)}\right)^{2}+2 c_{12} a_{2}^{(1)} a_{1}^{(2)}+c_{22}\left(a_{2}^{(1)}\right)^{2}\right] \\
& =\left(1-\alpha_{0}\right)\left[\frac{h_{1}^{2}}{g_{11}} \kappa_{1}^{2}+\frac{h_{2}^{2}}{g_{22}} \kappa_{2}^{2}-\alpha_{0} \frac{h_{1}^{2} h_{2}^{2}}{g_{11} g_{22}}\left(\kappa_{1}-\kappa_{2}\right)^{2}\right] \\
& =\left(1-\alpha_{0}\right)\left[\kappa_{1}^{2} \cos ^{2} \theta\left(X_{0}\right)+\kappa_{2}^{2} \sin ^{2} \theta\left(X_{0}\right)-\alpha_{0}\left(\kappa_{1}-\kappa_{2}\right)^{2} \cos ^{2} \theta\left(X_{0}\right) \sin ^{2} \theta\left(X_{0}\right)\right] \\
& =\left(1-\alpha_{0}\right) P\left(X_{0}\right)^{3} .
\end{aligned}
$$

So we obtain

$$
\mu_{2}=\lambda_{0}\left(1-\alpha_{0}\right)^{1 / 3} P\left(X_{0}\right)
$$

and

$$
\mu=\beta_{0} \varepsilon^{-2}+\lambda_{0}\left(1-\alpha_{0}\right)^{1 / 3} P\left(X_{0}\right) \varepsilon^{-4 / 3}+O\left(\varepsilon^{-1}\right) .
$$

To get the lowest eigenvalue, we choose $X_{0} \in \mathcal{P}$, i.e., $P\left(X_{0}\right)=P_{\min }$. Then we get

$$
\begin{aligned}
& \mu_{2}=\lambda_{0}\left(1-\alpha_{0}\right)^{1 / 3} P_{\min }, \\
& \mu=\beta_{0} \varepsilon^{-2}+\lambda_{0}\left(1-\alpha_{0}\right)^{1 / 3} P_{\min } \varepsilon^{-4 / 3}+O\left(\varepsilon^{-1}\right) .
\end{aligned}
$$

This ends our formal analysis.

\section{ACKNOWLEDGMENTS}

The author would like to thank Professor B. Helffer for communicating his preprints HM1, HM2 before publication. This work was partially supported by the National Natural Science Foundation of China, the Science Foundation of the Ministry of Education of China, and NUS Academic Research Grant R-146000-033-112. The main results of this paper were reported in the International Conference on Nonlinear PDE and Applications, held in Huangshan, China, June 2001; and the Concentration Phenomena and Vortex Dynamics Workshop, held in Pacific Institute for Mathematical Science, University of British Columbia, Canada, July 2001. The author would like to thank the organizers for the invitation.

\section{REFERENCES}

[A] S. Agmon, Lectures on exponential decay of solutions of second order elliptic equations: bounds on eigenfunctions of $N$-body Schrodinger operators, Princeton Univ. Press, NJ, 1982. MR 85f:35019

[A1] Y. Almog, Non-linear surface superconductivity for type II superconductors in the large domain limit, Arch. Ration. Mech. Anal., 165 (2002), 271-293. MR 2003k:82108

[BPT] P. Bauman, D. Phillips and Q. Tang, Stable nucleation for the Ginzburg-Landau system with an applied magnetic field, Arch. Rat. Mech. Anal., 142 (1998), 1-43. MR 99g:58040

[BS] A. Bernoff and P. Sternberg, Onset of superconductivity in decreasing fields for general domains, J. Math. Phys. 39 (1998), 1272-1284. MR 99a:82099 
[C] S. J. Chapman, Nucleation of superconductivity in decreasing fields, European J. Appl. Math., 5 (1994), part 1, 449-468; part 2, 469-494. MR 95m:82119

[CHO] S. J. Chapman, S. D. Howison and J. R. Ockendon, Macroscopic models for superconductivity, SIAM Review, 34 (1992), 529-560. MR 94b:82037

[DFS] M. del Pino, P. Felmer and P. Sternberg, Boundary concentration for eigenvalue problems related to the onset of superconductivity, Commun. Math. Phys., 210 (2000), 413-446. MR 2001k:35231

[dG] P. G. De Gennes, Superconductivity of Metals and Alloys, W. A. Benjamin, New York, 1966.

[DGP] Q. Du, M. Gunzburger and J. Peterson, Analysis and approximation of the GinzburgLandau model of superconductivity, SIAM Review, 34 (1992), 45-81. MR 93g:82109

[DH] M. Dauge and B. Helffer, Eigenvalues variation, I, Neumann problem for Sturm-Liouville operators, J. Differential Equations, 104 (1993), 243-262. MR 94j:47097

[G] G. P. Galdi, An Introduction to the Mathematical Theory of the Navier-Stokes Equations, vol. 1, Springer-Verlag, New York, 1994. MR 95i:35216a

[GL] V. Ginzburg and L. Landau, On the theory of superconductivity, in Collected Papers, Gordon and Breach, New York, 1967, 546-568. MR 38:5577

[GP] T. Giorgi and D. Phillips, The breakdown of superconductivity due to strong fields for the Ginzburg-Landau model, SIAM J. Math. Anal., 30 (1999), 341-359. MR 2000b:35235

[H] B. Helffer, Semi-Classical Analysis for the Schrödinger Operator and Applications, Lecture Notes in Mathematics, vol. 1336, Springer-Verlag, Berlin, 1988. MR 90c:81043

[HM1] B. Helffer and A. Morame, Magnetic bottles in connection with superconductivity, J. Functional Analysis, 185 (2001), 604-680. MR 2002m:81051

[HM2] B. Helffer and A. Morame, Magnetic bottles for the Neumann problem: the case of dimension 3, Proceedings of the Indian Academy of Sciences-Mathematical Sciences, 112 : (1) (2002), 71-84. MR 2003e:81048

[HP] B. Helffer and X. B. Pan, Upper critical field and location of surface nucleation of superconductivity, Ann. L'I.H.P. Analyse non Linéaire, 20 (2003), 145-181.

[J] H. Jadallah, The onset of superconductivity in a domain with a corner, J. Math. Phys., 42 (2001), 4101-4121. MR 2002k:82086

[JRS] H. T. Jadallah, J. Rubinstein and P. Sternberg, Phase transition curves for mesoscopic superconducting samples, Phys. Rev. Lett., 82 (1999), 2935-2938.

[L] O. A. Ladyzhenskaya, The Boundary Value Problems of Mathematical Physics, SpringerVerlag, New York, 1985. MR 87f:35001

[LP1] K. Lu and X. B. Pan, Gauge invariant eigenvalue problems in $\mathbb{R}^{2}$ and in $\mathbb{R}_{+}^{2}$, Trans. Amer. Math. Soc., 352 (2000), 1247-1276. MR 2000j:35248

[LP2] K. Lu and X. B. Pan, Eigenvalue problems of Ginzburg-Landau operator in bounded domains, J. Math. Phys., 40 (1999), 2647-2670. MR 2001c:35167]

[LP3] K. Lu and X. B. Pan, Estimates of the upper critical field for the Ginzburg-Landau equations of superconductivity, Physica D, 127 : (1-2) (1999), 73-104. MR 2000a:82075]

[LP4] K. Lu and X. B. Pan, Surface nucleation of superconductivity in 3-dimension, J. Differential Equations, 168 (2000), 386-452. MR 2002b:82069

[M] R. Montgomery, Hearing the zero locus of a magnetic field, Comm. Math. Phys., 168 (1995), 651-675, MR 96e:81044

[P1] X. B. Pan, Surface superconductivity in applied magnetic fields above $\mathrm{H}_{\mathrm{C}_{2}}$, Comm. Math. Phys., 228 (2002), 327-370. MR 2003i:82094

[P2] X. B. Pan, Upper critical field for superconductors with edges and corners, Calculus of Variations and PDE's, 14 (2002), 447-482. MR 2003f:82105

[P3] X. B. Pan, Superconducting thin films and the effect of de Gennes parameter, SIAM J. Math. Anal., 34 (2003), 957-991.

[P4] X. B. Pan, Superconductivity near critical temperature, J. Math. Phys., 44 (2003), 26392678.

[PK] X. B. Pan and K. H. Kwek, Schrödinger operators with non-degenerately vanishing magnetic fields in bounded domains, Trans. Amer. Math. Soc., 354 (2002), 4201-4227. MR 2003j:81052

[R] J. Rubinstein, Six lectures on superconductivity, in: Boundaries, Interfaces, and Transitions, M. Delfour ed., CRM Proceedings and Lecture Notes 13, Amer. Math. Soc., Providence, RI, 1998, pp. 163-184. MR 99e:82101 
[SdG] D. Saint-James and P. G. De Gennes, Onset of superconductivity in decreasing fields, Physics Letters, 6 : (5) (1963), 306-308.

[SS] E. Sandier and S. Serfaty, The decrease of bulk-superconductivity close to the second critical field in the Ginzburg-Landau model, SIAM J. Math. Anal., 34 (2003), 939-956.

[SST] D. Saint-James and G. Sarma and E. J. Thomas, Type II Superconductivity, Pergamon Press, Oxford, 1969.

[T] M. Tinkham, Introduction to Superconductivity, McGraw-Hill, New York, 1975.

Department of Mathematics, Zhejiang University, Hangzhou 310027, China - and Department of Mathematics, National University of Singapore, Singapore 119260

E-mail address: amaxbpan@dial.zju.edu.cn

E-mail address: matpanxb@nus.edu.sg 